ARTICLE

https://doi.org/10.1038/s41467-019-08977-6

\title{
Rapid active zone remodeling consolidates presynaptic potentiation
}

Mathias A. Böhme 1,2,3, Anthony W. McCarthy' ${ }^{1}$ Andreas T. Grasskamp (1) 1,2, Christine B. Beuschel ${ }^{2,3}$, Pragya Goel ${ }^{4}$, Meida Jusyte ${ }^{1}$, Desiree Laber ${ }^{5}$, Sheng Huang ${ }^{3}$, Ulises Rey ${ }^{3,6}$, Astrid G. Petzoldt ${ }^{3}$, Martin Lehmann ${ }^{1}$, Fabian Göttfert ${ }^{7}$, Pejmun Haghighi ${ }^{8}$, Stefan W. Hell ${ }^{7}$, David Owald ${ }^{5}$, Dion Dickman ${ }^{4}$, Stephan J. Sigrist ${ }^{2,3} \&$ Alexander M. Walter ${ }^{1}$

Neuronal communication across synapses relies on neurotransmitter release from presynaptic active zones (AZs) followed by postsynaptic transmitter detection. Synaptic plasticity homeostatically maintains functionality during perturbations and enables memory formation. Postsynaptic plasticity targets neurotransmitter receptors, but presynaptic mechanisms regulating the neurotransmitter release apparatus remain largely enigmatic. By studying Drosophila neuromuscular junctions (NMJs) we show that AZs consist of nanomodular release sites and identify a molecular sequence that adds modules within minutes of inducing homeostatic plasticity. This requires cognate transport machinery and specific AZscaffolding proteins. Structural remodeling is not required for immediate potentiation of neurotransmitter release, but necessary to sustain potentiation over longer timescales. Finally, mutations in Unc13 disrupting homeostatic plasticity at the NMJ also impair shortterm memory when central neurons are targeted, suggesting that both plasticity mechanisms utilize Unc13. Together, while immediate synaptic potentiation capitalizes on available material, it triggers the coincident incorporation of modular release sites to consolidate synaptic potentiation.

\footnotetext{
${ }^{1}$ Leibniz-Forschungsinstitut für Molekulare Pharmakologie (FMP), 13125 Berlin, Germany. ${ }^{2}$ NeuroCure Cluster of Excellence, Charité Universitätsmedizin, 10117 Berlin, Germany. ${ }^{3}$ Institute for Biology/Genetics, Freie Universität Berlin, 14195 Berlin, Germany. ${ }^{4}$ Department of Neurobiology, University of Southern California, Los Angeles, CA 90089, USA. ${ }^{5}$ Institut für Neurophysiologie, Charité Universitätsmedizin, 10117 Berlin, Germany. ${ }^{6}$ Department of Theory and Biosystems, Max Planck Institute of Colloids and Interfaces, Science Park Golm, 14424 Potsdam, Germany. ${ }^{7}$ Department of Nanobiophotonics, Max Planck Institute for Biophysical Chemistry, 37077 Göttingen, Germany. ${ }^{8}$ Buck Institute for Research on Aging, Novato, CA, USA. These authors contributed equally: Mathias A. Böhme, Anthony W. McCarthy. These authors jointly supervised this work: Stephan J. Sigrist, Alexander M. Walter. Correspondence and requests for materials should be addressed to S.J.S. (email: stephan.sigrist@fu-berlin.de) or to A.M.W. (email: awalter@fmp-berlin.de)
} 
$\mathrm{N}$ eurotransmitter-laden synaptic vesicles (SVs) release their content at presynaptic active zones (AZs) in response to $\mathrm{Ca}^{2+}$ influx through voltage gated channels that respond to action-potential (AP) depolarization. Neurotransmitter binding to postsynaptic receptors subsequently leads to their activation for synaptic transmission. Modulation of transmission strength is called synaptic plasticity. Long-term forms of synaptic plasticity are major cellular substrates for learning, memory, and behavioral adaptation $^{1,2}$. Mechanisms of long-term synaptic plasticity modify the structure and function of the presynaptic terminal and/or the postsynaptic apparatus. AZs are covered by complex scaffolds composed of a conserved set of extended structural proteins. ELKS/Bruchpilot (BRP), RIM, and RIM-binding protein (RBP) functionally organize the coupling between $\mathrm{Ca}^{2+}$-channels and release machinery by immobilizing the critical (M)Unc13 release factors in clusters close to presynaptic $\mathrm{Ca}^{2+}$-channels and thus generate SV release sites, at both mammalian and Drosophila synapses ${ }^{3-12}$. Whether and how discrete $\mathrm{AZ}$ release sites and the associated release machinery are reorganized during plastic changes remains unknown.

One crucial form of presynaptic plasticity is the homeostatic control of neurotransmitter release. This process, referred to as presynaptic homeostatic potentiation (PHP), is observed in organisms ranging from invertebrates to humans, but is perhaps best illustrated at the larval neuromuscular junction (NMJ) of Drosophila melanogaster ${ }^{13,14}$. Here, PHP requires the core AZscaffolding proteins RIM, RBP and Fife ${ }^{15-17}$ and physiologically coincides with the upregulation of SV release sites ${ }^{17,18}$. Yet it is unknown how these AZ-scaffolds mediate release site addition, which downstream molecules are needed for PHP, whether AZscaffold independent reactions occur, and whether these mechanisms extend to other forms of plasticity, e.g., during learning in the central nervous system.

Here, we combine genetic and electrophysiological analysis to reveal a molecular sequence that triggers structural remodeling of $\mathrm{AZ}$ scaffolding proteins (BRP/RBP), and which ultimately leads to Unc13 addition within minutes. Using super-resolution light microscopy, we identify a modular AZ nano-architecture built by these proteins (which correspond to SV release sites) that rapidly extends by incorporating additional modules for plasticity. This rapid remodeling critically depends on the core AZ scaffolding proteins $\mathrm{RBP} / \mathrm{BRP}$, but neither on the early AZ assembly factors Liprin- $\alpha / S y d-1$, nor on RIM or Fife. Additionally, AZ-remodeling was abolished in transport mutants previously shown to promote BRP/RBP transport. Strikingly, rapid addition of $\mathrm{AZ}$ nano-clusters was not required for the immediate expression of PHP on a minutes' timescale, but was essential to sustain potentiation thereafter. We identify Unc13A as a direct molecular target for PHP in experiments where Unc13A was delocalized from the AZ scaffolds. This mutant displayed sizable synaptic transmission but completely lacked PHP and AZremodeling. The same interference in mushroom body Kenyon cells of the Drosophila brain eliminated short-term memory, indicating that Unc13A is also a plasticity target in the central nervous system. In summary, we show that synapses capitalize on the available AZ material for immediate potentiation, but coincidently undergo release site addition via modular building blocks to consolidate stable synaptic potentiation. Thus, our work lays a foundation that will help to understand the mechanisms of a likely conserved presynaptic plasticity process that is important for dynamically adjusting and stabilizing neurotransmission across multiple timescales.

\section{Results}

Homeostatic plasticity regulates $\mathrm{AZ}$ protein levels. As a robust paradigm for assessing presynaptic plasticity over different time scales, we focused on PHP, which is well characterized at
Drosophila NMJs ${ }^{13}$. To induce plasticity on a timescale of minutes, postsynaptic ionotropic glutamate receptors were partially blocked using the non-competitive open-channel blocker Philanthotoxin-433 (PhTx) ${ }^{19}$ (Fig. 1a-d). This reduces postsynaptic sensitivity to neurotransmitter release from single SVs (reflected in a reduction of the amplitude of spontaneously occurring "minis", single SV fusion events, Fig. 1b). Initially, this also leads to a proportional decrease in AP-evoked transmission, but in under $10 \mathrm{~min}$, PHP increases the number of SVs released per AP (quantal content). This compensates for the postsynaptic interference, resulting in AP-evoked transmission comparable to baseline levels (Fig. 1b) ${ }^{19}$. To identify molecular adaptations during plasticity, we investigated whether the levels of any of the evolutionarily conserved AZ proteins were altered. We accordingly immunostained against BRP, RBP, Unc13A (we focused on Unc13A, the Unc13 isoform dominating evoked SV release at Drosophila NMJ synapses ${ }^{4}$; flybase: unc-13-RA), Syx-1A, Unc18, and Syd-1 (as motoneuronally expressed Syd-1-GFP) (Fig. 1c; Supplementary Fig. 1a). In agreement with previous observations ${ }^{18,20}$, we found that 10 min of PhTx treatment increased AZ BRP-levels by about $50 \%$ (Fig. 1c, d). In addition, we found that RBP, Unc13A, and Syx-1A increased by about 30\%,60\%, and $65 \%$, respectively (Fig. 1c, d). The AZ levels of $\mathrm{RBP} / \mathrm{BRP}$, Unc13A/BRP, and Syx-1A/BRP scaled proportionally over all AZ sizes (Supplementary Fig. 1b; Ctrl, black lines). This proportionality was preserved upon PhTx treatment (Supplementary Fig. 1b; PhTx, blue lines). Notably, the AZ-levels and distribution of the essential Sec1/(M)Unc18 family protein Unc18-which was recently found to function in $\mathrm{PHP}^{21}$-were unaffected (Fig. 1c, d), demonstrating specific up-regulation of a subset of AZ proteins. Another AZ protein, the assembly factor Syd-1, even displayed a slight reduction upon PhTx (Supplementary Fig. 1a), further underscoring a high degree of specificity.

To verify that these AZ-adaptations were specific to functional glutamate receptor interference, and to address their relevance over a longer time window, we investigated larvae bearing mutations in a glutamate receptor subunit (Fig. 1e-h). Deletion of the high-conductance receptor subunit IIA (GluRIIA) resulted in a similarly reduced postsynaptic sensitivity to single SV fusion events (Fig. 1f) as PhTx treatment (Fig. 1b). Because under these circumstances PHP also increases quantal content to achieve similar AP-evoked transmission (Fig. 1f), gluRIIA mutants have extensively been used to investigate long-term PHP (over the $3-4$ days of larval development) 22,23 . Immunostainings against BRP, RBP, Unc13A, and Syx-1A confirmed their (in this case larger) elevation on this longer timescale (compare Fig. 1g, h with 1c, d) $(100 \%, 70 \%, 400 \%$ and $200 \%$, respectively, compared to $50 \%, 30 \%, 60 \%$, and $65 \%$ upon PhTx treatment). Unlike the stoichiometric increase observed for BRP/Unc13A within minutes, this long-term PHP revealed enhanced Unc13A AZincorporation (Supplementary Fig. 1c). Another distinction was a remarkable reorganization and eight-fold increase of Unc18 (Fig. 1g, h; Supplementary Fig. 1d, e). Our data hence imply that considerable $\mathrm{AZ}$ restructuring occurs within minutes of $\mathrm{PHP}$ induction, which is further enhanced across longer-lasting timescales.

Rapid addition of release site modules during PHP. We next sought to investigate how the altered levels of AZ proteins during PHP affected their nanoscopic topology by super-resolution STED microscopy $(x-y$ resolution $\sim 30-40 \mathrm{~nm})$. As noted before, planar AZs revealed clearly distinguishable individual Unc13A/ BRP/RBP spots arranged in a ring-like geometry ${ }^{4-7}$ (Fig. 2a). It was recently shown by single molecule imaging that these individual clusters likely contain several (probably few tens of) 


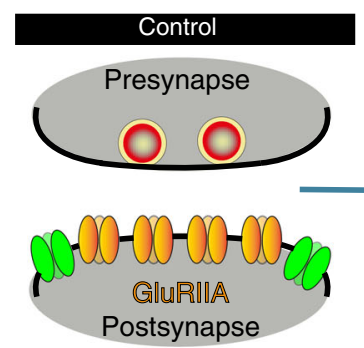

b
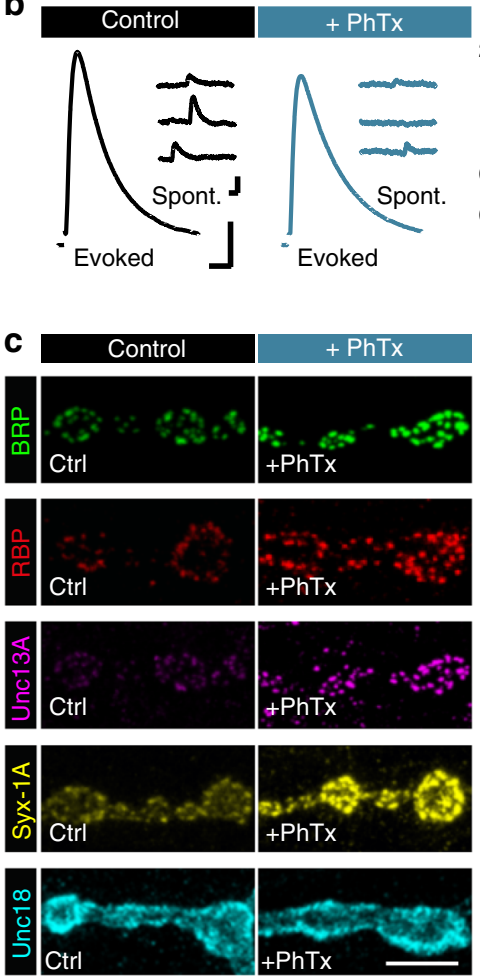
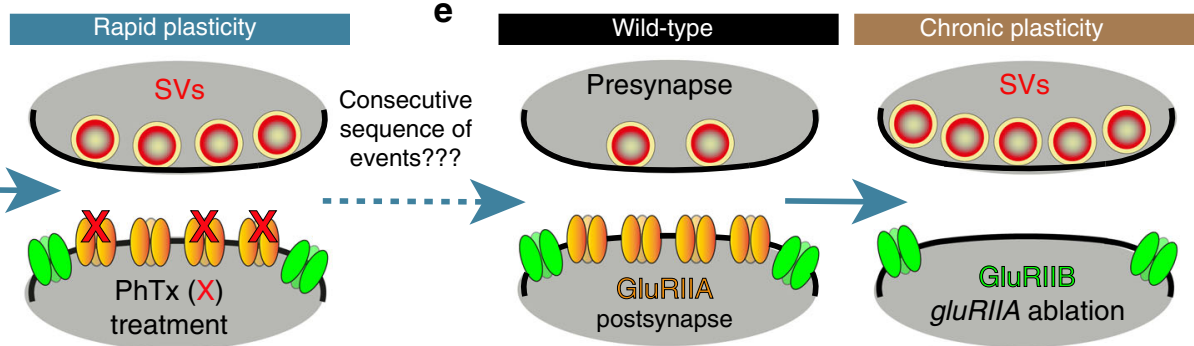

f
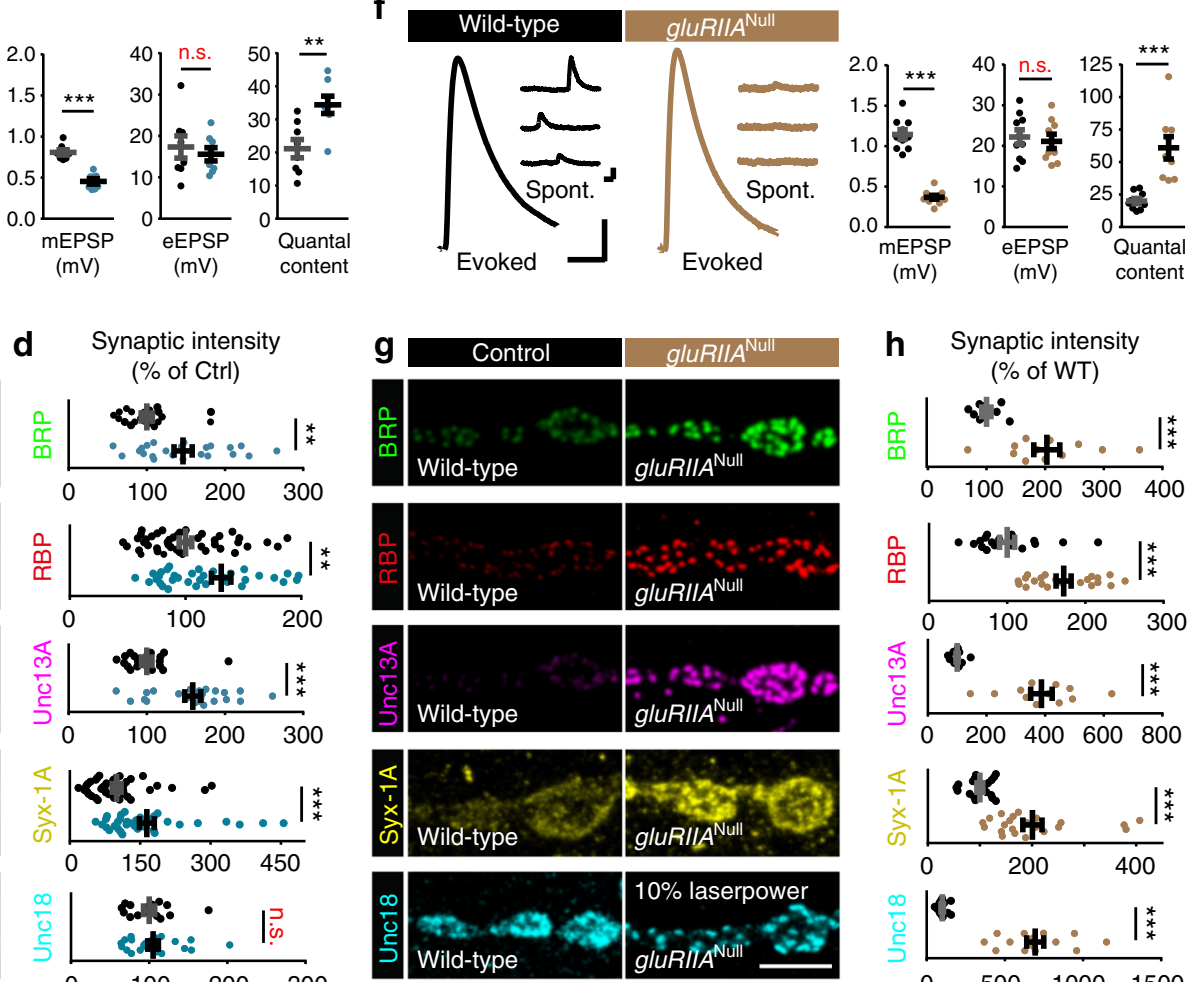

Fig. 1 Rapid homeostatic plasticity regulates AZ protein levels. a Sketch of investigated conditions for rapid plasticity: control synapses (left) are compared with rapid plasticity (10 min of PhTx (red "X"); right). Rapid plasticity increases the number of SVs released (red). b, $\mathbf{f}$ Representative traces of eEPSP (evoked), mEPSP (spont.) and their quantification in Ctrl (black) and PhTx (blue) treated (b) or wild-type (black) and gluRIlA Null (brown) (f) cells. c, $\mathbf{g}$ NMJs labelled with indicated antibodies in Ctrl (black) and PhTx (blue) treated (c) or in wild-type (black) and gluRllA Null (brown) (g) animals. d, $\mathbf{h}$ Quantification of BRP, RBP, Unc13A, Syx-1A, and Unc18 AZ-levels in \% of Ctrl in Ctrl (black) and PhTx (blue) treated (d) or in \% of wild-type (WT) in wildtype (black) and gluRIIANull (brown) (h) animals. e Sketch of investigated conditions for chronic plasticity: wild-type synapses (left) are compared with gluRIIANull mutants. Chronic plasticity greatly increases the number of SVs released. See also Supplementary Fig. 1. Source data as exact normalized and raw values, detailed statistics including sample sizes and $P$ values are provided in the Source Data file. Scale bars: b, f eEPSP: 25 ms, 5 mV; mEPSP: 50 ms, 1 $\mathrm{mV} ; \mathbf{c}, \mathbf{g} 5 \mu \mathrm{m}$. Statistics: Student's unpaired T-test was used for comparisons in (b) and ((f) eEPSP) and Mann-Whitney $U$ test for all other comparisons. ${ }^{\star \star} P \leq 0.01 ;{ }^{\star \star \star} P \leq 0.001 ;$ n.s., not significant, $P>0.05$. All panels show mean \pm s.e.m.

molecules in the case of $\mathrm{BRP}^{24}$. We detected the number of clusters per AZ in single AZ-images, which was largely heterogeneous for all three proteins, with a simple peak detection algorithm (Fig. 2c-e (black bars)). However, in all cases the cluster number per AZ increased upon PhTx-treatment (Fig. 2c-e (blue bars)), and slightly increased further in gluRIIANull mutants (Supplementary Fig. 2a; brown bars). With increasing AZ-cluster number the $\mathrm{AZ}$ diameter (measured from the $\mathrm{AZ}$ center to the center of the clusters) also increased in both conditions (Supplementary Fig. 2b, c), consistent with previous STED analysis performed on BRP-rings ${ }^{18}$. Notably, the observed remodeling only affected cluster numbers, and did not alter their intensities (Supplementary Fig. 2b, c). Consequently, the first conclusion of our analysis is that PHP increases the number of Unc13A/BRP/ RBP nano-clusters per AZ within minutes.
We also wanted to investigate whether the overall AZ-topology changed upon cluster incorporation. Notably, averaging of STEDimages was recently used to generate a three-dimensional model of an average synapse, displaying the mean protein localization at high resolution ${ }^{25}$. Thus, to compare the overall single AZtopology, AZ images were centered, sorted by the number of clusters, aligned by rotation, and averaged. Two different alignment methods were used. In the first procedure, images were simply rotated such that the cluster with the highest intensity was positioned at the top (Supplementary Fig. 3a and Methods for details). Even though this procedure only targeted a single pixel per image (the position of the brightest cluster), the remaining (lower intensity) clusters were often found in similar relative positions, such that averaging revealed a simple polygonal geometrical series (Supplementary Fig. 3a), demonstrating some 

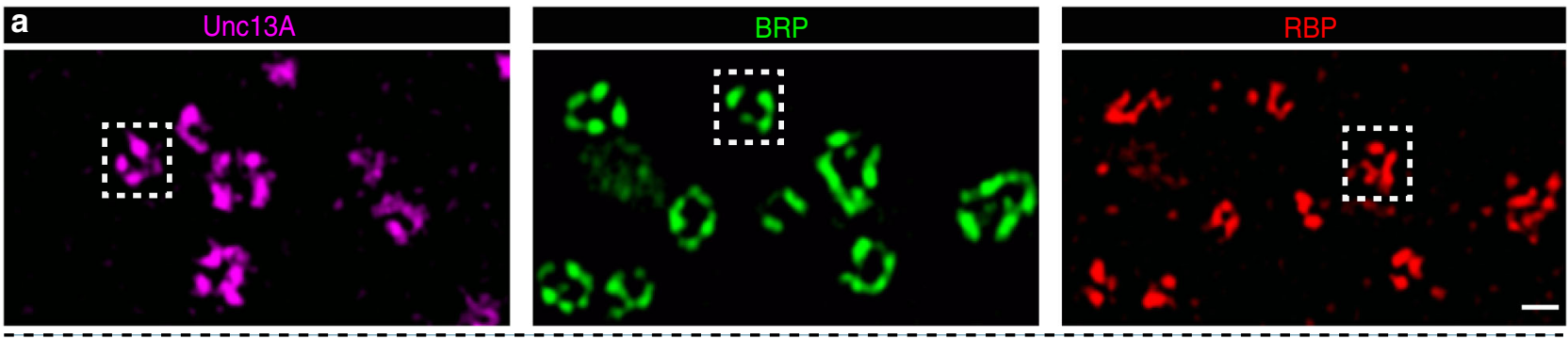

b

Synaptic cluster numbers in wild-type exemplified for Unc13A

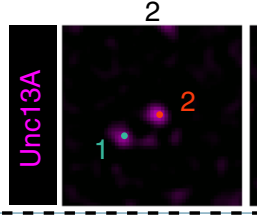

3
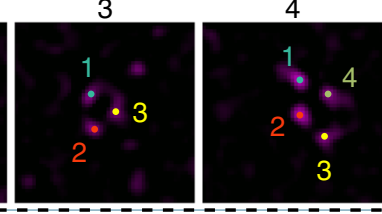

6
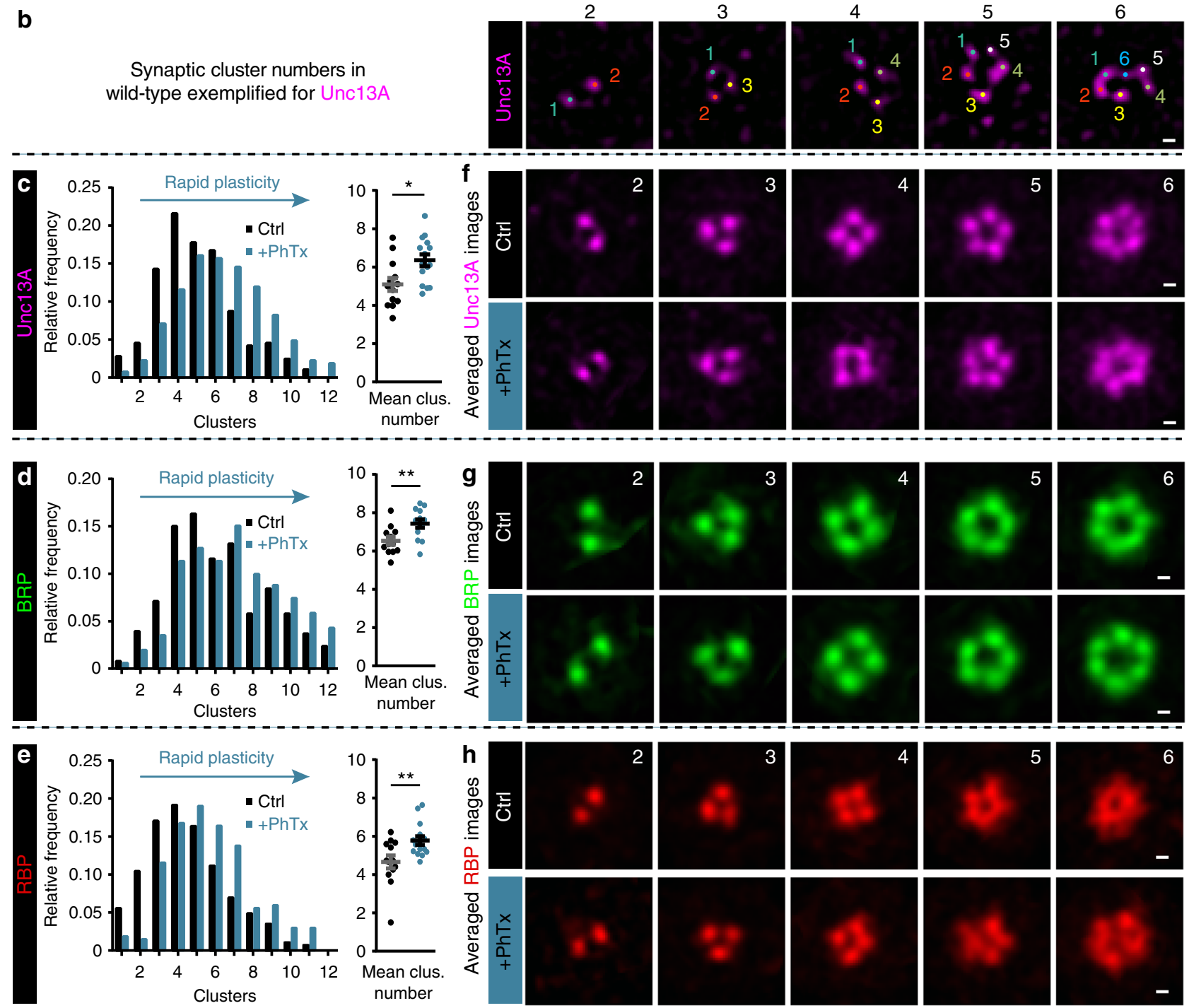

Fig. 2 Rapid plasticity alters Unc13A/BRP/RBP AZ protein cluster number. a STED microscopy images containing several AZs with variable numbers of protein clusters of Unc13A (magenta), BRP (green) and RBP (red). Dashed white boxes mark one AZ example used for the cluster number counting. b Example AZs with 2-6 Unc13A clusters, marked by colored dots and used for cluster number counting. (c-e, left) Frequency distribution of Unc13A (c), BRP (d), and RBP (e) modules per AZ either without (Ctrl, -PhTx; black) or with PhTx (+PhTx; blue) treatment. f-h Average of rotated STED images stained against Unc13A (f), BRP (g), and RBP (h) with 2-6 modules either without (Ctrl) or with PhTx (+PhTx) treatment. See also Supplementary Figs. 2-4. Source data as exact values, detailed statistics including sample sizes and $P$ values are provided in the Source Data file. Scale bars: a $200 \mathrm{~nm}$; $(\mathbf{b}, \mathbf{f}-\mathbf{h}) 50 \mathrm{~nm}$. Statistics: Mann-Whitney $U$ test. ${ }^{\star} P \leq 0.05 ;{ }^{\star \star} P \leq 0.01$. Panels (c-e) show NMJ-wise means of $A Z$-mean \pm s.e.m.

regularity. In a more refined analysis, we simultaneously considered the position of all clusters per AZ (Supplementary Fig. 3c and Methods for details) which also revealed a simple geometrical pattern for Unc13A, BRP, and RBP (Fig. 2f-h and Supplementary Fig. 3c). This stereotypical arrangement was best seen for AZs containing two to six clusters but less clear for AZs containing more than that (which could mean that these are less regular; Supplementary Fig. 2d, e). This arrangement was unaltered upon
PhTx-treatment or gluRIIA ablation (Fig. 2f-h and Supplementary Fig. $4 \mathrm{a}-\mathrm{c})$. Notably, the results of these averaging approaches did not necessarily result in specific patterns, as neither random categorization of the single $\mathrm{AZ}$ images nor applying this methodology to Syx-1A and Unc18 (which are diffusely distributed at the AZ) resulted in regular but instead in highly irregular/ random fluorescence patterns (Supplementary Figs. $3 \mathrm{~b}$ and $2 \mathrm{f}, \mathrm{g}$ ). This also demonstrates that structural features are only conserved 

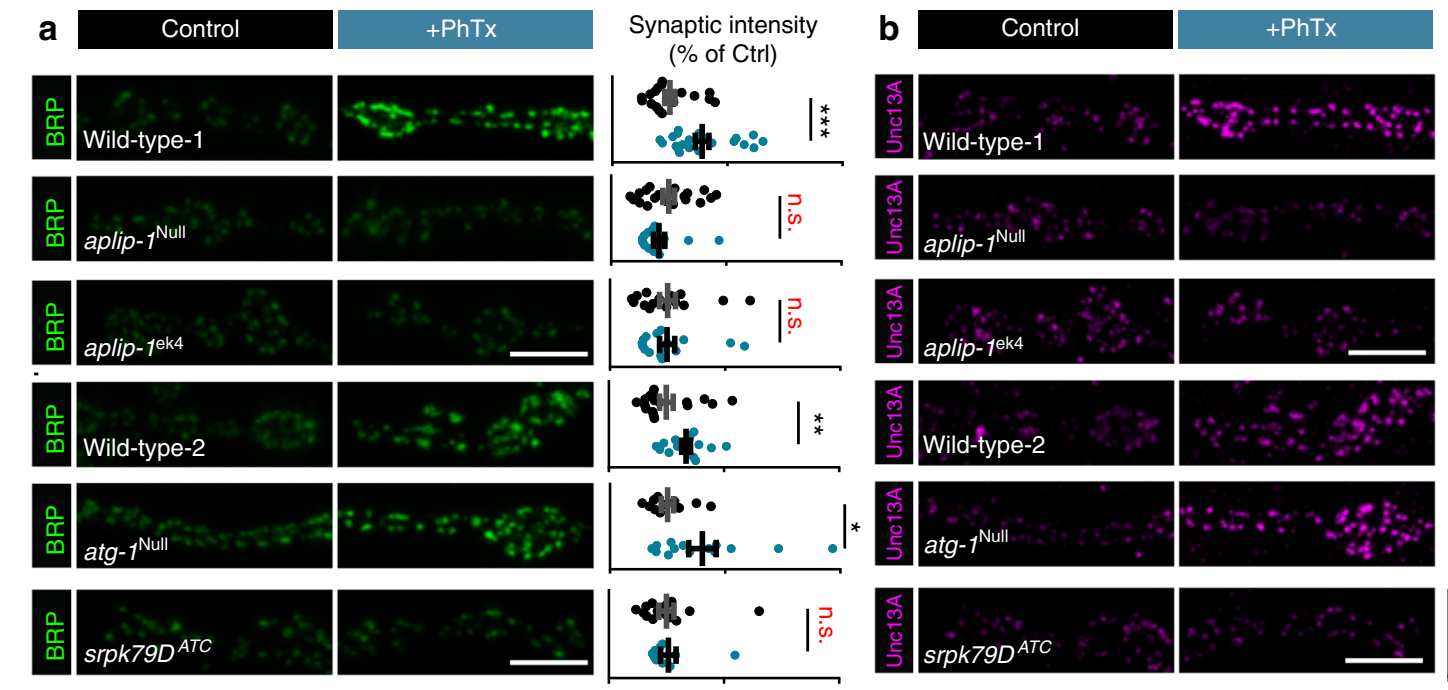

Synaptic intensity (\% of Ctrl)
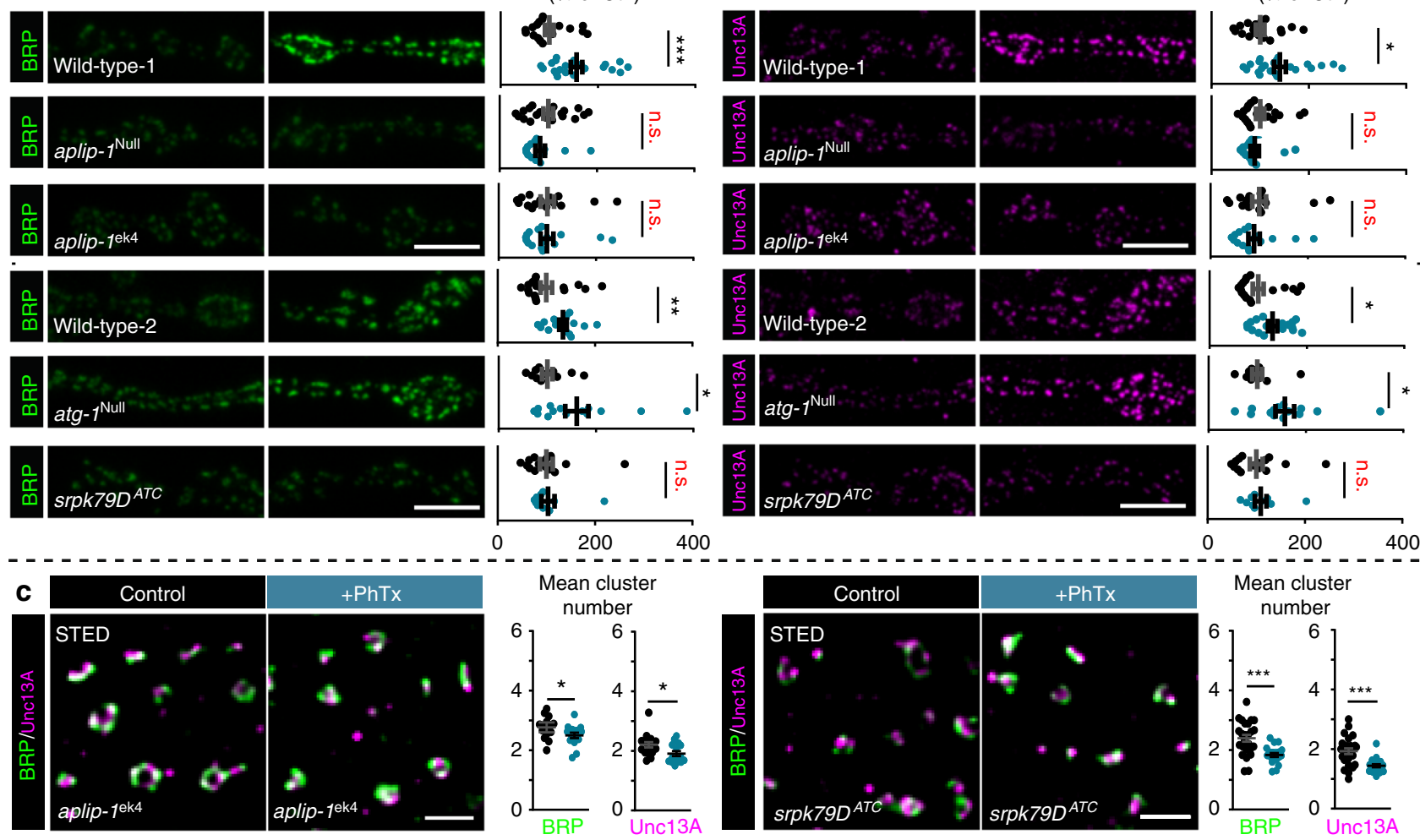

Fig. 3 Rapid AZ-remodeling during PHP requires Aplip-1 and Srpk79D. a, b Muscle 4 NMJs of segment A2-A5 from third instar larvae and quantification of synaptic levels of wild-type-1, aplip-1Null, aplip-1ek4, wild-type-2, atg1 and srpk79DATC labelled with the indicated antibodies without (Ctrl; black) and with 10 min of PhTx (+PhTx; blue) treatment. Two independent experiments were performed, wild-type-1 was used as control for aplip- $7^{\text {Null }}$ and aplip- $7^{e k 4}$ while wild-type-2 was used for atg 7 Null and srpk79DATC. c Average BRP and Unc13A cluster number per AZ either without (Ctrl, -PhTx; black) or with PhTx (+PhTx; blue) treatment in aplip-7ek4 and srpk79DATC. See also Supplementary Figs. 5, 6 and Movie 1. Source data as exact normalized and raw values, detailed statistics including sample sizes and $P$ values are provided in the Source Data file. Scale bars: a, b $5 \mu$ m; c $500 \mathrm{~nm}$. Statistics: Mann-Whitney $U$ test. ${ }^{\star} P \leq 0.05 ;{ }^{\star \star} P \leq 0.01 ;{ }^{\star \star \star} P \leq 0.001 ;$ n.s., not significant, $P>0.05$. All panels show mean \pm s.e.m.

across AZs containing the same number of clusters. It should be noted that only the average images (Fig. 2f-h; Supplementary Fig. 4) depend on this procedure, while detecting the effects of PhTx or gluRIIA ablation on cluster numbers (Fig. 2c-e; Supplementary Fig. 2a) was fully independent of this.

Thus, these findings imply that complexes of the core AZscaffold form discrete nano-modular structures, which correspond to SV release sites, and that rapid presynaptic plasticity triggers their fast AZ-incorporation which is even further enhanced over longer-timescales.

Impairing BRP/RBP transport disrupts rapid AZ-remodeling. The remarkable remodeling of $\mathrm{AZ}$ material within the short timeframe of PhTx-treatment (minutes) raised the question of how this is mechanistically achieved. We first considered whether local presynaptic protein translation could be required ${ }^{26}$. However, treatment of larvae with $50 \mu \mathrm{g} / \mathrm{ml}$ of the translation blocker cycloheximide (prior and during PhTx-treatment) did not disrupt structural remodeling of BRP or vGlut (vesicular glutamate transporter) at AZs (Supplementary Fig. 5a), consistent with remodeling being translation-independent. Moreover, the functional increase in quantal content remained expressed in the presence of the blocker (Supplementary Fig. 5b), consistent with previous reports ${ }^{19,20}$.

Because active kinesin-dependent protein transport is required for long-term homeostatic plasticity in gluRIIA Null mutants ${ }^{27}$, we asked whether BRP/RBP transport mechanisms might be employed for AZ remodeling. For this, we investigated proteins involved in $\mathrm{BRP} / \mathrm{RBP}$ transport by their mutation which causes abnormal BRP/RBP accumulation in the moto-neuronal axons, such as Atg1 (Unc-51) ${ }^{28}$, serine-arginine (SR) protein kinase at location 79D (Srpk79D ${ }^{29,30}$ ), and App-like interacting protein (Aplip-1, Jip1 or JNK interacting protein in mammals), a selective RBP transport-adaptor ${ }^{31}$ (Fig. 3a, b). While we observed clear PhTx-induced BRP-/Unc13A-upscaling in wild-type controls as well as in atg1 mutants (Fig. 3a, b), remodeling was fully absent

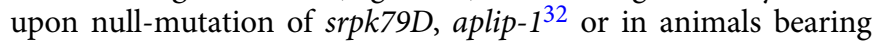
an Aplip-1 point mutation that selectively prevents kinesin light chain interaction (aplip-1 $\left.{ }^{e k 4}\right)^{33}$ (Fig. 3a, b). Additionally, STED microscopy revealed that aplip-1 $1^{e k 4}$ and srpk79D ${ }^{A T C}$ mutants appeared to contain fewer BRP/Unc13A clusters per $\mathrm{AZ}$ on average than wild-type (compare Fig. 2c, d with Fig. $3 c$ ) and fully lost the capacity to increase cluster numbers upon PhTxtreatment (even a decrease was observed, Fig. 3c). We also discovered that upon motoneuronal Aplip-1 or Srpk79D knockdown, Unc13A-GFP co-accumulated with aberrant axonal BRP aggregates (Supplementary Fig. 5c, d). Interestingly, a partial coaccumulation of BRP/Unc13A-GFP (but to a lower extent in comparison to the knock-downs) was also present in the control situation, in line with an at least partial co-transport that we occasionally observed in live-imaging experiments (Supplementary Fig. 5c-g; Movie 1).

We further wanted to elaborate on the involvement of active protein transport during $\mathrm{PhTx}$-induced AZ-remodeling by interfering with the cytoskeletal tracks used for short-range 
a
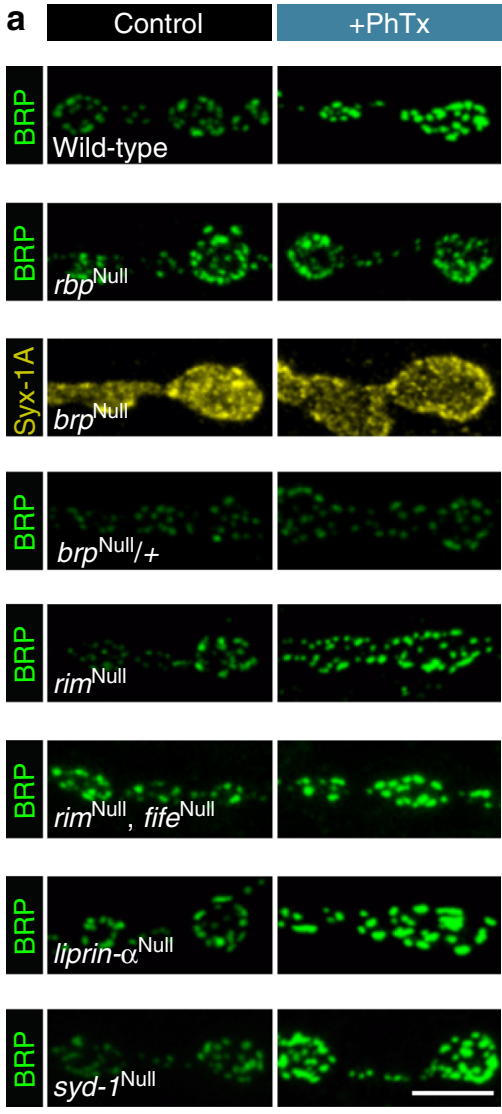

Synaptic intensity (\% of Ctrl)
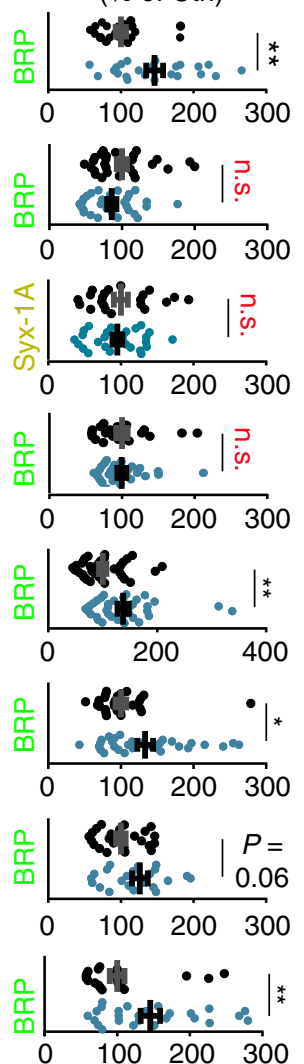
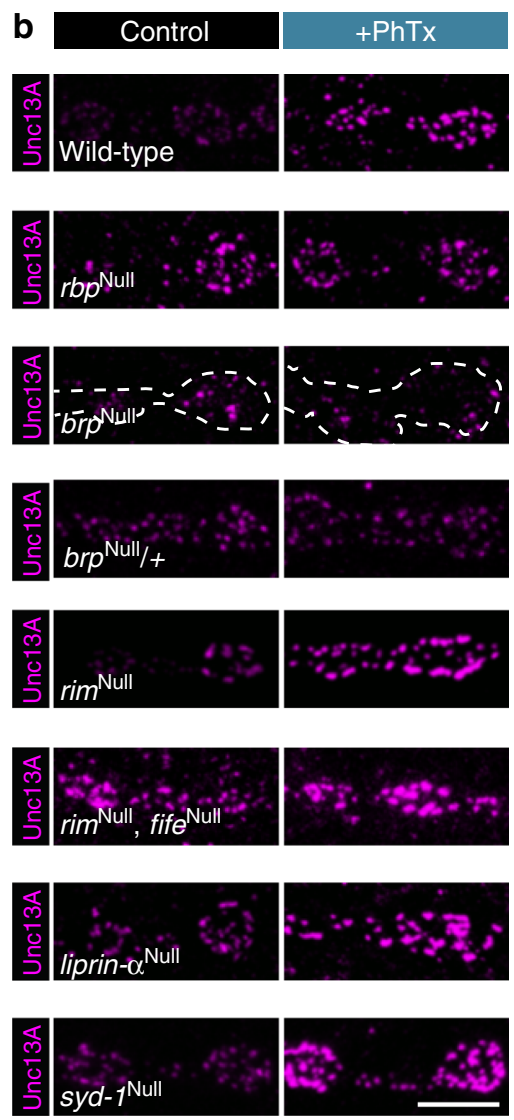

Synaptic intensity (\% of Ctrl)
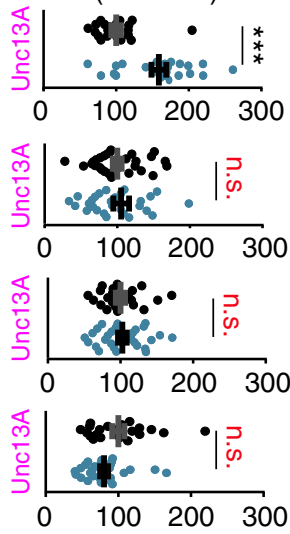

$\begin{array}{llll}0 & 100 \quad 200 \quad 300\end{array}$
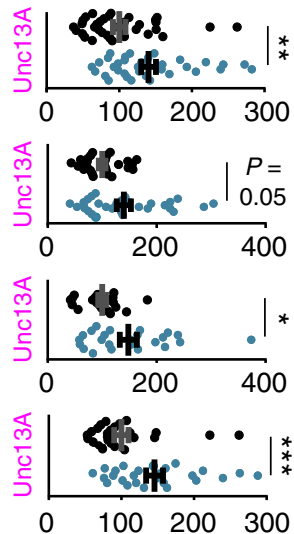

Fig. 4 Rapid AZ-remodeling during PHP requires BRP and RBP. a, b Confocal images and quantification of synaptic intensities of muscle 4 NMJs of abdominal segment 2-4 from third instar larvae at wild-type, rbp Null, brp Null, brp Null/,+ rim $^{\text {Null }}$, rim ${ }^{\text {Null }} / f i f e^{\text {Null, }}$, liprin- $\alpha^{\text {Null, }}$, and syd $7^{\text {Null }}$ NMJs labelled with the indicated antibodies without (control; black) and with $10 \mathrm{~min} \mathrm{PhTx} \mathrm{(+PhTx;} \mathrm{blue)} \mathrm{treatment.} \mathrm{For} \mathrm{wild-type,} \mathrm{images} \mathrm{and} \mathrm{data} \mathrm{were} \mathrm{adapted} \mathrm{and} \mathrm{replotted}$ from Fig. 1. Please note, although wild-type controls were performed in parallel to every mutant genotype to check for functional AZ-remodeling upon PhTx-treatment in each set of experiments, we do not show all WT-control here due to space limitations. Therefore, AZ-protein levels should not be compared between genotypes. Source data as exact normalized and raw values (also of additional Wild-type controls for genotypes where PhTx-treatment failed to induce AZ-remodeling), detailed statistics including sample sizes and $P$ values are provided in the Source Data file. Scale bars: $5 \mu$ m. Statistics: Mann-Whitney $U$ test. ${ }^{\star} P \leq 0.05$; ${ }^{\star \star} P \leq 0.01 ;{ }^{\star \star} P \leq 0.001$; n.s., not significant, $P>0.05$. All panels show mean \pm s.e.m.

transport using Latrunculin B (an actin polymerization blocker). While the AZ levels of BRP/Unc13A were already slightly enhanced by Latrunculin B treatment (Supplementary Fig. 6a, b), $\mathrm{PhTx}$-treatment failed to induce the typical increase of the AZ levels of these proteins (in fact a reduction was observed, Supplementary Fig. 6a, b). Thus, the actin cytoskeleton and active $\mathrm{BRP} / \mathrm{RBP}$ transport are required for rapid $\mathrm{AZ}$ remodeling.

Rapid homeostatic AZ-remodeling depends on BRP and RBP. We next investigated which of the evolutionarily conserved core AZ scaffolding proteins are required for rapid AZ-remodeling. Loss of RBP fully blocked the rapid, PhTx-induced increase of BRP/Unc13A (Fig. 4a, b). BRP was also essential, because the typical increase in Syx-1A and Unc13A observed upon PhTx treatment was abolished (Compare Fig. 4a, b with Fig. 1c, d). Notably, BRP-amounts appear to be rate-limiting because PhTxinduced AZ-remodeling was blocked in larvae heterozygous for a brp null allele $\left(b r p^{\mathrm{Null}} /+\right)$ (Fig. $\left.4 \mathrm{a}, \mathrm{b}\right)$. In contrast, null mutation of RIM, which abolished $\mathrm{PHP}^{17}$, did not interfere with AZremodeling (Fig. 4a, b). Furthermore, the simultaneous deletion of RIM and Fife (a possible RIM homologue which is required for $\mathrm{PHP}^{15,34}$ ) did not interfere with AZ-remodeling. Thus, RIM and Fife appear to act downstream of BRP/RBP and are non-essential for AZ-remodeling (Fig. 4a, b).
$\mathrm{AZ}$ assembly is initiated by the conserved scaffolding proteins Liprin- $\alpha$ and Syd-1, which both regulate AZ size ${ }^{35-39}$. We reasoned that $\mathrm{AZ}$ growth - as observed here during plasticitymay capitalize on the same molecular machinery as de novo AZ formation, and therefore tested whether BRP/Unc13A-scaling depended on those proteins. However, liprin- $\alpha^{\text {Null }}$ and $s y d-1^{\text {Null }}$ mutants revealed normal PhTx-induced BRP/Unc13A-scaling (Fig. $4 \mathrm{a}, \mathrm{b}$ ), indicating that these factors are dispensable. Thus, systematic investigation of evolutionarily conserved AZ scaffolding proteins reveals a selective dependence on the core AZscaffolds BRP and RBP for structural remodeling during plasticity.

AZ-remodeling is required for chronic -but not rapid- PHP. Several studies have shown that presynaptic release positively correlates with AZ-size $7,40-43$. Therefore, we expected that the increase of AZ-BRP/Unc13A observed upon PhTx treatment would functionally increase presynaptic release (Fig. 1a-d). However, it is not entirely obvious whether the AZ-remodeling (which continues beyond the minutes' timescale during long-term PHP (Fig. 1e-h)) would be essential for rapid PHP. For instance, loss of RBP was shown to occlude both AZ remodeling (Fig. 4) and the functional increase in quantal content ${ }^{16}$, suggesting a pivotal role in both adaptations. Yet PHP and AZ-remodeling do 


\section{a}

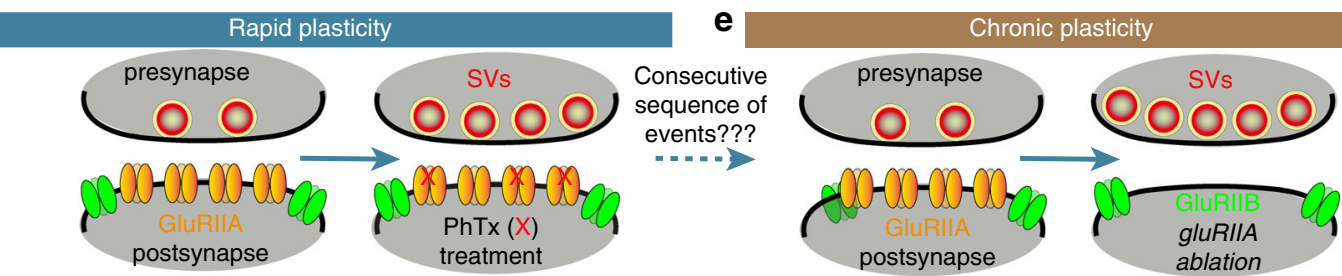

b

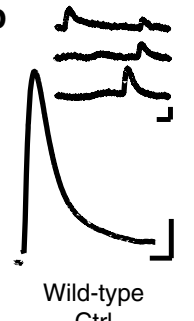

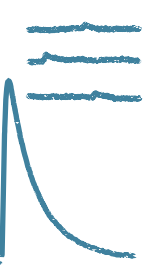

Wild-type

$\mathrm{PhTx}$
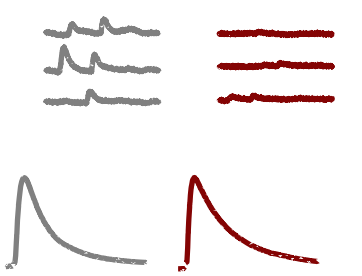

brp Null

Ctrl

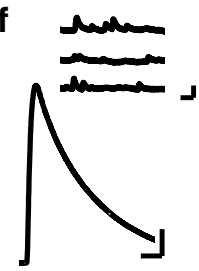

brp Null $+P h T x$

C
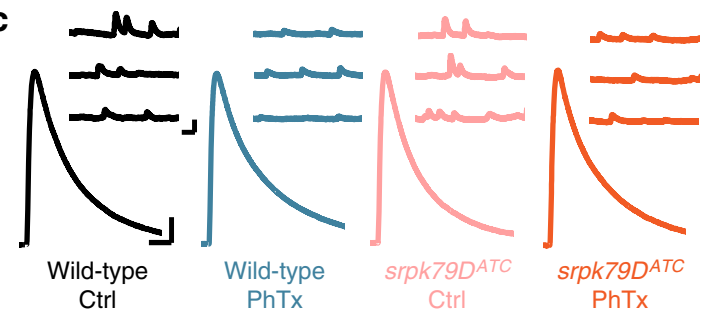

srpk79D ${ }^{A T C}$

PhTx
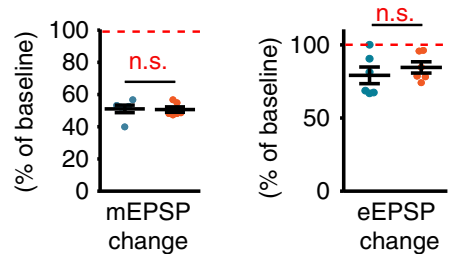

- Wild-type + PhTx

srpk79D ${ }^{A T C}+\mathrm{PhTx}$
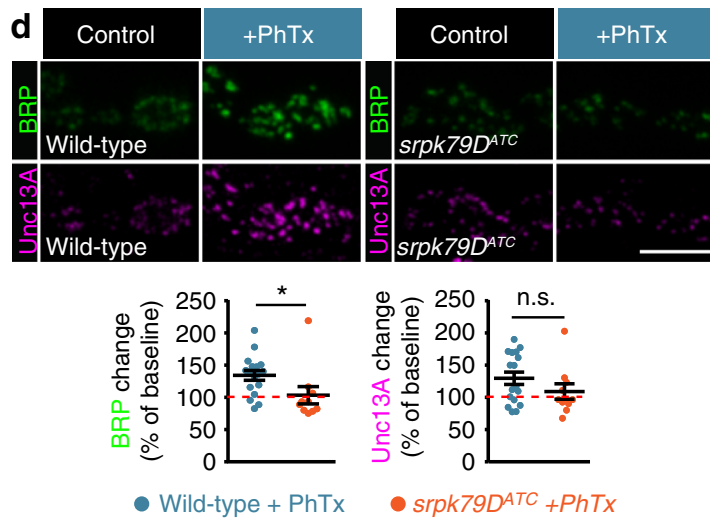

not go hand-in-hand in the case of RIM (and Fife) mutants, whose AZs remodel, but which cannot express PHP (increased quantal content ${ }^{15,17}$ ). These observations prompted us to systematically investigate the relevance of AZ-remodeling for the rapid induction and sustained expression of PHP.
Wild-type

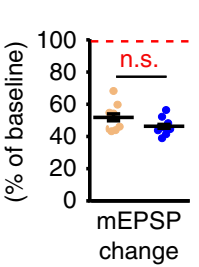

ghroHAN
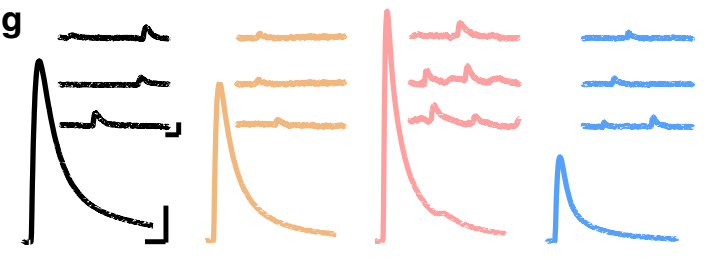

Wild-type
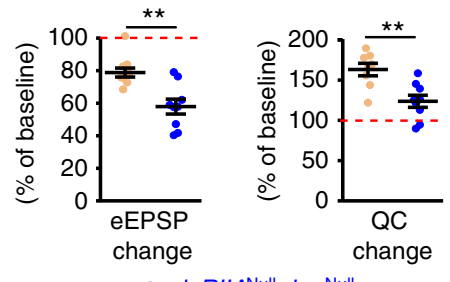

gluRIIA $A^{\text {Null } ; ~ b r p ~ N u l l ~}$

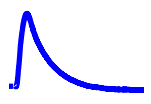

$\underset{\text { gluRIIANull: }}{\operatorname{brp} \text { Null }}$

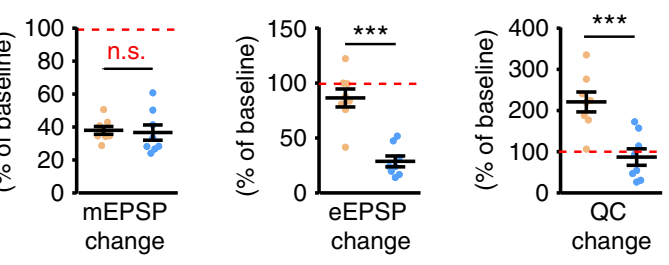

- gluRIIANull gllIANull; srpk79DATC
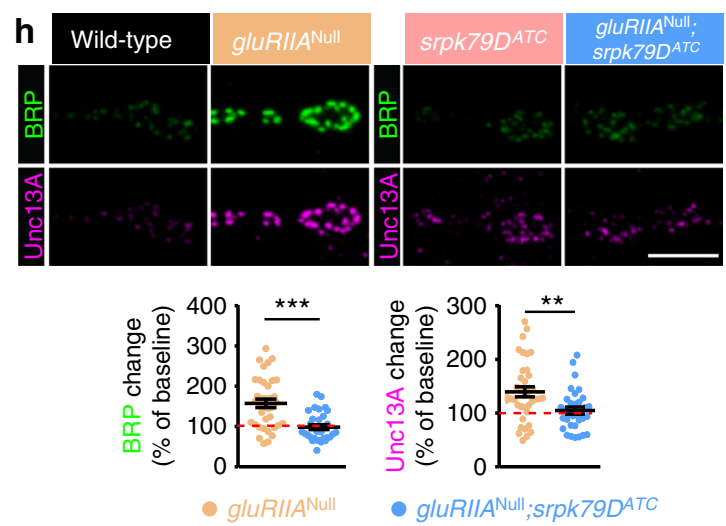

We first investigated the dependence of rapid PHP on the rapid

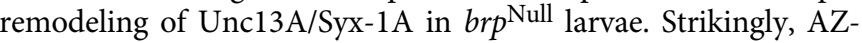
remodeling was blocked (Fig. 4a, b), but functional PHP expression (an increased quantal content) persisted at levels comparable to the wild-type/control situation (Fig. 5a, b). In 
Fig. 5 AZ-remodeling sustains NT-release potentiation over longer timescales. a Sketch of investigated conditions for rapid plasticity: control synapses (left) are compared with rapid plasticity (10 min of PhTx (red " $\mathrm{X}$ "); right). Rapid plasticity increases the number of SVs released (red). b, c (left) Representative traces of eEPSP (evoked) and mEPSP (spont.) of the indicated genotypes with and without PhTx-treatment. (Right) Quantifications of percentage change of mEPSP amplitude, eEPSP amplitude and quantal content (QC) upon PhTx-treatment. Values are divided by the corresponding measurement in the absence of PhTx for each genotype (dashed red line corresponds to 100\%/no change). d (left) Confocal images of muscle 4 NMJs of abdominal segment 2-5 from third instar larvae at wild-type and srpk79DATC NMJs labelled with the indicated antibodies without (control; black) and with 10 min PhTx (+PhTx; blue) treatment. (Right) Quantification of percentage change of synaptic BRP and Unc13A levels in wild-type (blue) and srpk79DATC (orange) upon PhTx-treatment compared to baseline of control treatment for each genotype (dashed red line). Data are modified from Fig. 2. e Sketch of investigated conditions for chronic plasticity: wild-type synapses (left) are compared with gluRIIANull mutants. Chronic plasticity greatly increases the number of SVs released. $\mathbf{f}, \mathbf{g}$ Same as in $(\mathbf{b}, \mathbf{c})$ but compared to baseline of each control genotype. $\mathbf{h}$ Same as in (d) but compared to baseline fluorescence values of wild-type for gluRIIA Null and srpk79D ${ }^{A T C}$ for gluRIIANull;srpk79D ${ }^{A T C}$. See also Supplementary Fig. 7. Source data as exact normalized and raw values, detailed statistics including sample sizes and $P$ values are provided in the Source Data file. See also Supplementary Figure 10 and 11 for nonnormalized values. Scale bars: eEPSP: $25 \mathrm{~ms}, 5 \mathrm{mV}$; mEPSP: $50 \mathrm{~ms}, 1 \mathrm{mV} ; \mathbf{d}, \mathbf{h} 5 \mu \mathrm{m}$. Statistics: Student's unpaired $T$-test was used for comparisons in ((b) quantal content change), (f), (g) and Mann-Whitney $U$ test for all other comparisons. ${ }^{\star} P \leq 0.05 ;{ }^{\star \star} P \leq 0.01 ;{ }^{\star \star \star} P \leq 0.001 ;$ n.s., not significant, $P>0.05$. All panels show mean \pm s.e.m.

addition, mutants of Srpk79D (whose AZs also do not undergo PhTx-induced AZ-remodeling; Fig. 3a, b and replotted in Fig. 5d) were likewise able to increase their quantal content (Fig. 5c), showing that $\mathrm{AZ}$ remodeling can be uncoupled from rapid $\mathrm{PHP}$ expression. Thus, even though AZ-remodeling occurs on a similar time-scale, it is not required to rapidly enhance the quantal content in these cases.

We next investigated whether elevation of the AZ protein levels was required to consolidate the increased quantal content over chronic time-scales in gluRIIA Null mutants (Fig. 5e-h). Indeed, PHP was severely impaired in $b r p^{\text {Null }}$, gluRIIA Null double mutants (Fig. 5f). In an otherwise wild-type background, the increase in quantal content (upon gluRIIA-null mutation) was much larger than when BRP was additionally deleted (Fig. 5f). We could ensure that the impairment was not due to the overall reduced release in $b r p^{\text {Null }}$ mutants, as a loss to compensate for the gluRIIA ablation was also seen in $\operatorname{srpk} 79 D^{A T C}$ mutants (which had synaptic transmission comparable to wild-type cells (Fig. 5g), no AZremodeling upon PhTx-treatment (Fig. 3a, b), intact PHP upon PhTx-treatment (Fig. 5c) and severely impaired PHP expression in gluRIIA Null (Fig. 5g)). Importantly, AZ-remodeling was also fully blocked in gluRIIA Null, srpk79D ${ }^{A T C}$ double mutants (Fig. 5h). An intermediate behavior was seen in the case of the aplip-1 $1^{k 4}$ mutant, (Supplementary Fig. 7), possibly because other transport adapters might compensate in this situation. Together, this suggests that $\mathrm{PHP}$ rapidly increases neurotransmitter release through modulation of the available AZ components, but in addition immediately induces AZ-remodeling to ensure its consolidation.

Presynaptic potentiation requires Unc13A. We next sought to identify the molecular substrate of PHP. Previous experiments established a requirement for the al subunit of the voltage gated $\mathrm{Ca}^{2+}$-channel Cacophony $(\mathrm{Cac})^{19,44}$. In line with this, the levels of $\mathrm{Cac}$ as well as the $\mathrm{Ca}^{2+}$-influx increase upon $\mathrm{PhTx}$ treatment (Fig. 6a, b) ${ }^{44,45}$. We furthermore investigated Unc13A. A slight PhTx-induced BRP-/RBP-scaling persisted upon Unc13A loss, but was weaker than in the wild-type situation (Fig. 6a, b), possibly due to slightly elevated BRP/RBP-AZ-levels already in the nonPhTx-treated $u n c 13 A^{\text {Null }}$ situation ${ }^{4}$. Notably, Cac-levels were still increased, even to a slightly larger extent than in the wild-type situation (Fig. 6a, b). However, functional PHP, the increase in quantal content, was completely lost (Fig. 6c, d). This indicates that Unc13A-like RIM and RBP ${ }^{16,17}$ - plays an essential role in the plastic enhancement of NT release during PHP.

Rapid PHP and learning rely on the Unc13A N-terminus. The observation that Unc13A is essential for PHP is fully consistent with the previous findings that RIM and RBP are required ${ }^{16,17}$, because these proteins likely function in Unc13A AZ recruitment and activation (see discussion). As in other species ( $M$. musculus/C. elegans), this interaction depends on the (M)Unc13 $\mathrm{N}$-terminus ${ }^{4,46-50}$. To investigate the functional relevance of the Unc13A N-terminus for rapid PHP and AZ-remodeling, we used an Unc13A mutant lacking the $\mathrm{N}$-terminal AZ-localization sequence (named C-term-GFP; Fig. 6e), which uncouples Unc13A from the central BRP/RBP scaffold ${ }^{7}$ (and therefore supposedly also uncouples SV fusion from a possible regulatory function of RIM - see discussion). Importantly, the magnitude of AP-evoked synaptic transmission in these mutants was largely restored compared to the detrimental effect of $u n c 13^{\text {Null }}$ mutation (compare Fig. 6h (C-term-GFP; grey traces) with Fig. 6h (full-length Unc13A-GFP; black traces) and ref. ${ }^{7}$ ). However, in contrast to control larvae (unc13 ${ }^{\text {Null }}$ with full-length Unc13A-GFP rescue; Fig. 6f-i), C-term-GFP mutants (unc13 ${ }^{\text {Null }}$ with C-term-GFP rescue; Fig. 6f-i) completely lacked AZ-remodeling (Fig. 6f, g) (note that BRP levels were already enhanced in the non-PhTxtreated group, Supplementary Fig. 8a, b), Furthermore, unlike in control larvae, no rescue of evoked transmission and no increase in quantal content was seen upon PhTx treatment, indicating that C-term mutants were deficient of functional PHP (Fig. 6h-i). This demonstrates a dependence of PHP on the Unc13A N-terminus.

Although synapses vary tremendously in their excitability, input/output relationship, and transmitter type, the presynaptic release machinery is remarkably conserved in most systems and across species ${ }^{9}$. We wondered whether the principles of rapid presynaptic adaptation of the peripheral nervous system might be utilized in other synapse types and in other forms of plasticity, like the ones involved in learning and memory formation. Because short-term memory functions on timescales comparable to the PhTx-induced rapid homeostatic plasticity ${ }^{51}$, we investigated whether the Unc13A C-term mutant also exhibited learning deficits.

We specifically expressed C-term-GFP either alone, or while simultaneously knocking down endogenous full-length Unc13A (Unc13A-RNAi), in mushroom body Kenyon cells (KCs). KC output synapses undergo learning-induced plasticity and are required for the formation of short-term memories ${ }^{52}$. Knockdown of endogenous Unc13A and expression of C-term-GFP were confirmed using antibodies against GFP (labelling C-termGFP but not endogenous protein) and the Unc13A N-terminus (labelling the endogenous protein but not C-term-GFP where the N-terminal epitope is deleted) (Fig. 7a). Additionally, we confirmed the strong efficacy of the Unc13A-RNAi via Western blot (knock-down in the entire brain using the pan-neuronal elav-Gal4 driver; Supplementary Fig. 9a). 

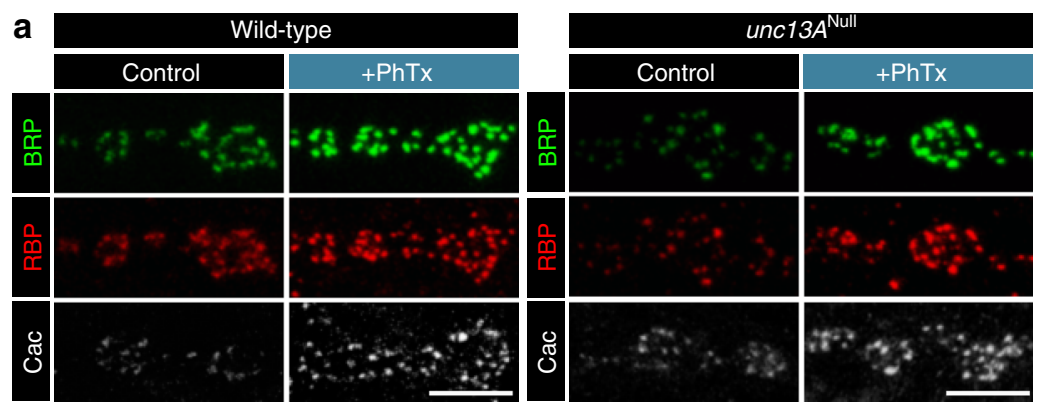

b BRP change
(\% of baseline)

RBP change

(\% of baseline)

Cac change

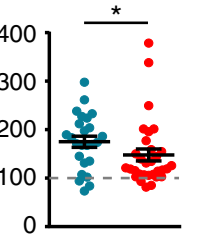

Wild-type +PhTx
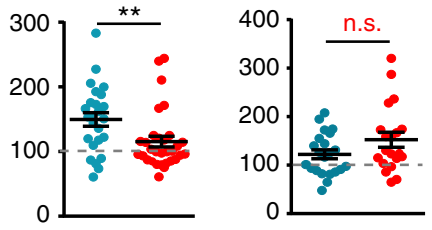

- unc13A $A^{\text {Null }}+$ PhTx

C

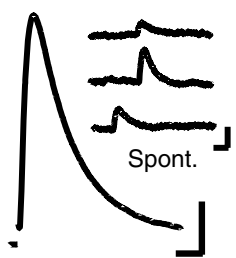

Evoked

Wild-type

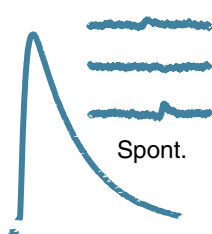

Evoked

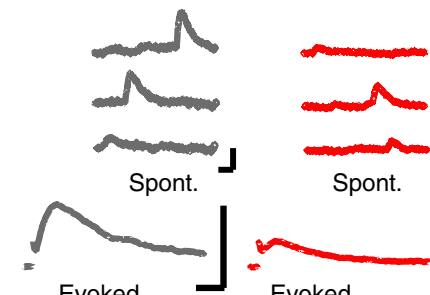

Evoked

unc13A $A^{\text {Nul }}$

Evoked

Wild-type +PhTx d

mEPSP change (\% of baseline)

eEPSP change

(\% of baseline)

(\% of baseline)
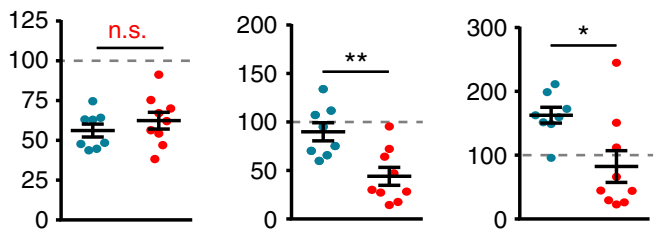

QC change

Wild-type + PhTx

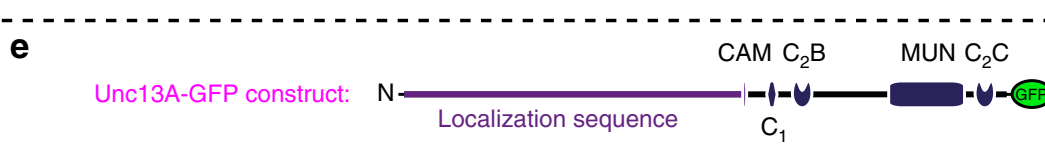

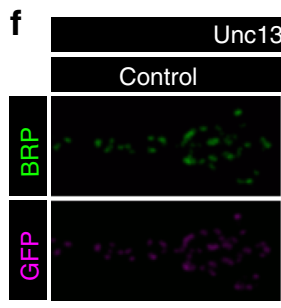

Unc13A-GFP

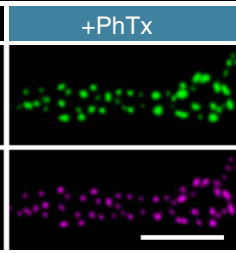

h

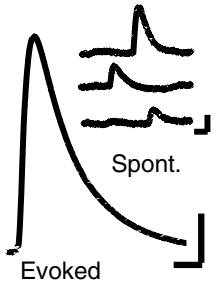

Unc13A-GFP

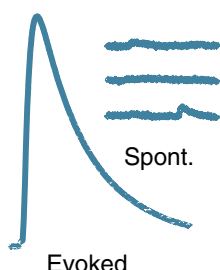

Evoked

Unc13A-GFP $+\mathrm{PhTx}$
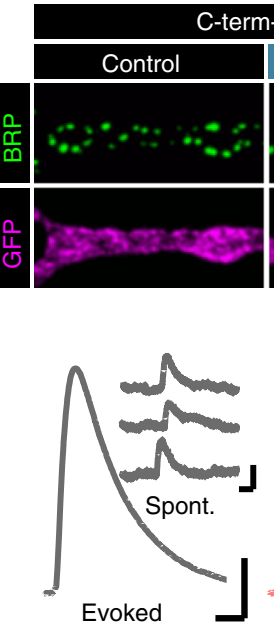

C-term-GFP
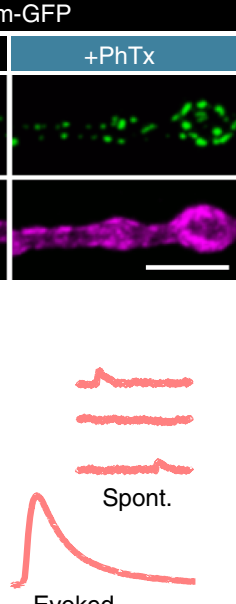

C-term-GFP

C-term-GFP construct:

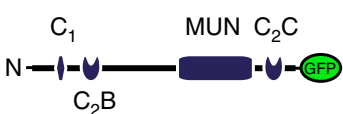

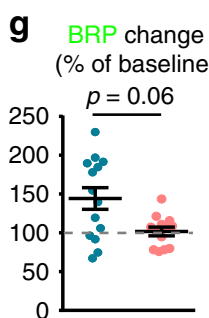

i mEPSP change (\% of baseline)

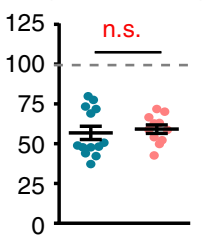

GFP change

(\% of baseline)

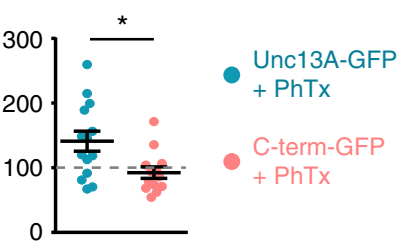

\section{eEPSP change}

QC change

(\% of baseline)
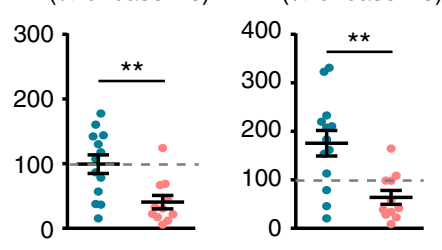

Fig. 6 Unc13A and its N-terminus are critical for rapid PHP and AZ-remodeling. a Confocal images of muscle 4 NMJs of abdominal segment 2-5 from 3rd instar larvae at wild-type (left) and unc13ANull (right) NMJs labelled with the indicated antibodies without (control; black) and with 10 min PhTx (+PhTx; blue) treatment. b Quantification of percentage change of synaptic BRP, RBP and Cac AZ-levels in wild-type (blue) and unc13ANull (red) upon PhTxtreatment compared to the same measurement in the absence of PhTx for each genotype (dashed grey line indicates 100\%/no change). c Representative traces of eEPSP (evoked) and mEPSP (spont.) in wild-type and unc $13 A^{\text {Null }}$ animals without (Ctrl; black or grey) and with 10 min PhTx (+PhTx; blue or light red) treatment. d Quantifications of percentage change of mEPSP amplitude, eEPSP amplitude and quantal content (QC) in PhTx-treated wild-type (blue) and unc13A Null (light red) cells compared to the same measurement obtained without PhTx for each genotype. Traces for (c) were replotted from Fig. 1. e Left: Full-length Unc13A construct used in rescue experiments of unc13 Null animals. Functional domains for AZ localization, Calmodulin- (CAM), lipidbinding (C1, C2B, C2C) and the MUN domain relevant for SV release are shown. Right: Schematic of Unc13A construct lacking the N-terminal localization sequence (C-term-GFP rescue). f-i Same as in (a-d) for cells re-expressing Unc13A-GFP (blue) or C-term-GFP (light red) in the unc13Null background. See also Supplementary Figure 8. See also Supplementary Figure 10 for non-normalized values. Source data as exact normalized and raw values, detailed statistics including sample sizes and $P$ values are provided in the Source Data file. Scale bars: a, f $5 \mu$ m; c, $\mathbf{h}$ eEPSP: $25 \mathrm{~ms}, 5 \mathrm{mV} ;$ mEPSP: $50 \mathrm{~ms}$, $1 \mathrm{mV}$. Statistics: Student's unpaired T-test was used for comparisons in ((d) mEPSP, eEPSP change), ((i) mEPSP change) and Mann-Whitney $U$ test for all other comparisons. ${ }^{\star} P \leq 0.05 ;{ }^{\star \star} P \leq 0.01$; n.s., not significant, $P>0.05$. All panels show mean \pm s.e.m. 


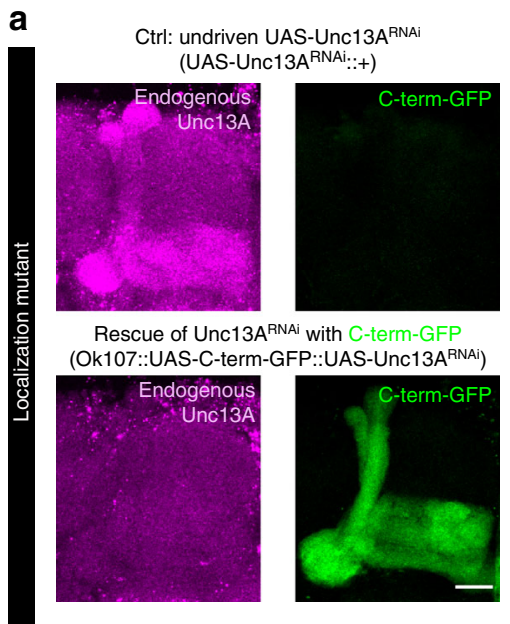

b

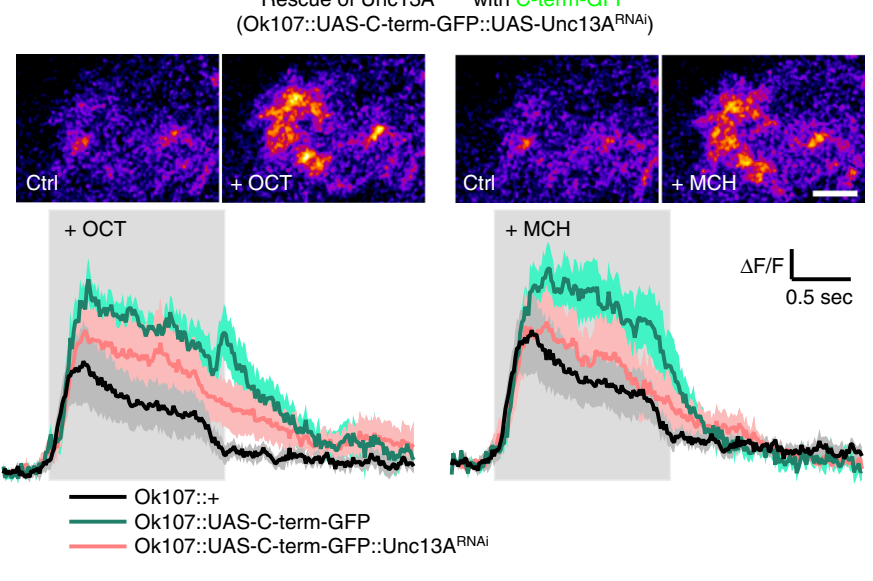

c

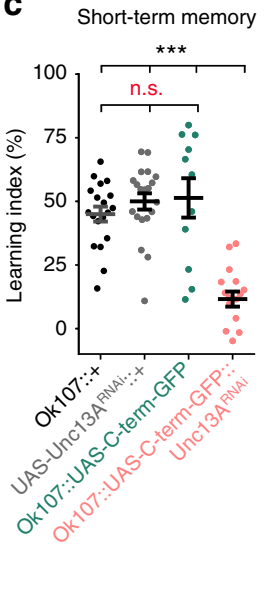

Fig. 7 Mutants incapable of PHP impair short-term memory. a Confocal images of adult Drosophila mushroom-body regions of control (top; undriven UAS-

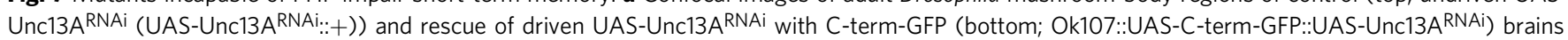
labelled with the indicated antibodies. b Averaged odor responses measured at the level of presynaptic boutons of $\mathrm{M} 4 / 6$ ( $\mathrm{MBON}-\beta^{\prime} 2 \mathrm{mp} / \mathrm{MBON}-\gamma 5 \beta^{\prime} 2 \mathrm{a} /$ MBON- $\beta 2 \beta^{\prime} 2 a$ ) mushroom body output neurons (compare ref. 54 or ref. ${ }^{89}$ ). Above: sample images of two-photon recordings from $M 4 / 6$ cells (20 frames averaged respectively) before and after odor onset. Grey shading indicates the time at which the odor was applied. Left panels OCT and right MCH response. Below: averaged odor responses. Five responses per odor were averaged per animal. Solid lines show mean responses ( $n=5-6$ animals per genotype). Shaded areas represent the SEM. c Short-term memory scores after mushroom body-specific C-term-GFP rescue after Unc13A downregulation via locally driven RNAi expression (Ok107::UAS-C-term-GFP::Unc13ARNAi) compared to controls expressing the driver, but not the RNAi (Ok107::+), the RNAi without driver (UAS-Unc13ARNAi::+) or mushroom body-specific overexpression of the C-term-GFP construct (Ok107::UAS-C-term-GFP). See also Supplementary Fig. 9. Source data as exact raw values, detailed statistics including sample sizes and P values are provided in the Source Data file. Scale bars: a $20 \mu$ m; $\mathbf{b} 10$ $\mu \mathrm{m}$. Statistics: nonparametric one-way analysis of variance (ANOVA) test, followed by a Tukey's multiple comparison test. ${ }^{\star \star \star} P \leq 0.001 ;$ n.S., not significant, $P>0.05$. All panels show mean \pm s.e.m. For representative images experiments were repeated twice with at least $6-7$ brains per genotype

Because the C-term-GFP construct largely rescued the detrimental effect of unc13 null mutation at the $\mathrm{NMJ}^{7}$, we predicted that general transmission from KCs should be functional for both the C-term-GFP and the C-term-GFP/ Unc13A-RNAi conditions. To verify this, we performed in vivo two photon $\mathrm{Ca}^{2+}$-imaging (GCaMP6f) experiments in adult flies to assess odor-evoked responses at the M4/6 (MBON- $\beta^{\prime} 2 \mathrm{mp} /$ MBON- $\left.\gamma 5 \beta^{\prime} 2 \mathrm{a} / \mathrm{MBON}-\beta 2 \beta^{\prime} 2 \mathrm{a}\right)$ mushroom body output neurons, a postsynaptic circuit element directly downstream of $\mathrm{KCs}^{53,54}$. Indeed, robust $\mathrm{Ca}^{2+}$ transients in response to odor stimulation were observed in all genetic constellations tested (Fig. 7b). We conclude as a result that $\mathrm{KC}$ output synapses expressing $\mathrm{C}$-termGFP or C-term-GFP/Unc13A-RNAi are functional under naive conditions. Together with the finding that naive odor avoidance was not statistically different between all these groups (Supplementary Fig. 9b), this allowed us to test whether either condition would interfere with learning and memory.

We assessed short-term memory in adult Drosophila using classical aversive olfactory conditioning. Flies were trained by pairing an odor with an electric shock and learning was scored by subsequently assaying avoidance of that odor ${ }^{55}$. All control groups showed similarly robust memory performance, while the relevant C-term-GFP/Unc13A-RNAi mutant (where endogenous Unc13A is knocked down and replaced by the C-term mutant incapable of PHP (Fig. 6e-i)) showed severe short-term memory impairments (Fig. 7c). Whether these impairments are indeed a consequence of the loss of a similar plasticity mechanism as the one observed at the NMJ or whether they are also related to differences in the synaptic transmission profile (e.g., short-term plasticity which is also affected in this mutant ${ }^{7}$ ) remains to be established. Nevertheless, our data clearly indicate that Unc13A is a target for similar forms of plasticity (Unc13A-RNAi knockdown also impaired short-term memory, Supplementary Fig. 9b, c). Thus, our data imply that structural, functional, and behavioral adaptations are linked and that different forms of presynaptic plasticity may converge on Unc13A.

\section{Discussion}

Synapses are able to modify their transmission strength by undergoing plastic changes. This synaptic plasticity is crucial for neuronal circuit adaptation including learning and memory processes $^{1,56}$. Molecular mechanisms for postsynaptic plasticity have been defined in considerable detail ${ }^{2}$. However, presynaptic mechanisms also modulate transmission strength in many synapse types and species ${ }^{13,51,57}$. Homeostatic plasticity is a wellstudied form of presynaptic plasticity at the Drosophila NMJ where an enhancement of AP-evoked neurotransmitter release counterbalances decreased postsynaptic receptor sensitivity. A number of relevant signaling molecules and pathways, including BMP signaling, CaMKII signaling, TOR signaling, proteasomal degradation, and trans-synaptic signaling are required for this ${ }^{13,19,58-60}$. These factors appear to converge on two principal avenues to enhance presynaptic transmitter release, via increased $\mathrm{Ca}^{2+}$ channel amounts and AP-induced $\mathrm{Ca}^{2+}$ influx ${ }^{44,45,61}$ and secondly via an increase in the number of releasable SVs and their associated release sites $17,18,61$. Nevertheless, some conditions were observed where $\mathrm{Ca}^{2+}$ influx or $\mathrm{Ca}^{2+}$-channel levels were increased but the quantal content was not (Fig. 6a-d and ref. ${ }^{17}$ ), suggesting that release site addition or activation is a required contributor. On the minutes' time-scale, structural AZremodeling was observed, yet whether and how this contributes to the enhancement of NT-release remained unclear ${ }^{18}$.

In the present study, we uncover a sequence of presynaptic molecular events that mediate AZ-remodeling (Fig. 8). We identify the presynaptic cytomatrix as a highly dynamic structure that can add discrete nano-modules of core proteins within minutes. In the initial phase of this structural remodeling, RBP 


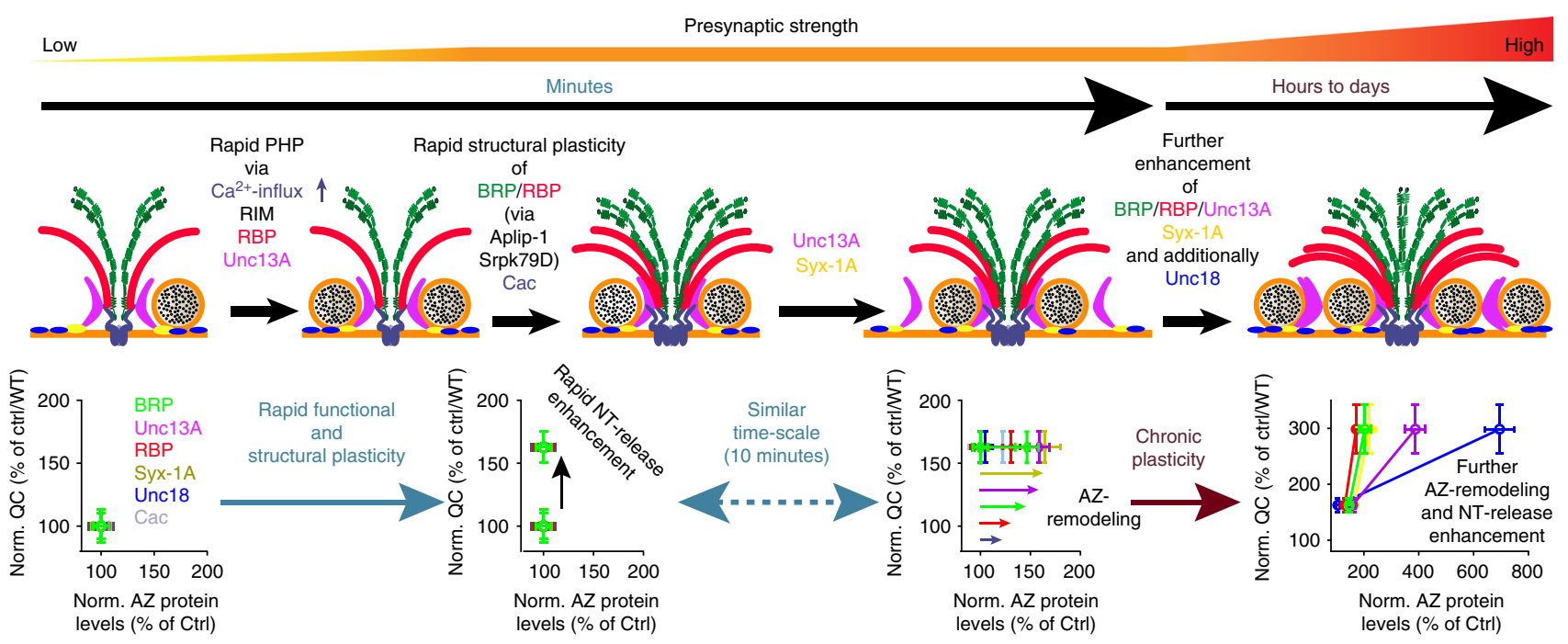

Fig. 8 Sequence of events enabling rapid and sustained homeostatic plasticity Top row: Illustration of AZ modes addressed. Bottom row: Plot of normalized quantal content (QC) vs. AZ protein levels of experiments performed in Fig. 1 normalized to either Ctrl ( - PhTx) for rapid plasticity or wild-type for chronic plasticity. In the basal activity mode (left), BRP (green), RBP (red), Syx-1A (yellow), Unc18 (blue) and Unc13A (magenta) provide two SV release sites at the $\mathrm{Ca}^{2+}$-channel (Cac; light blue). However just one release site is active (occupied by SV). (Second left) During the rapid functional plasticity phase the quantal content (and thus neurotransmitter (NT) release) is rapidly enhanced within minutes via mechanisms involving altered $\mathrm{Ca}^{2+}$-influx as well as RIM, RBP and Unc13A. On a comparable time-scale (minutes), BRP and RBP are incorporated in a pre-existing AZ in an Aplip-1/Srpk79D dependent manner and additionally Cac-levels also increase (third cartoon). The BRP/RBP incorporation enhances AZ levels of Unc13A/Syx-1A providing an additional release sites (fourth left). This rapid structural AZ-remodeling is not required for the rapid functional plasticity but directly acts on the consolidation of the release enhancement. (Right) On longer time-scales, chronic plasticity then further enhances the AZ-levels of BRP, RBP, Syx-1A in a conserved stoichiometry, while Unc13A and Unc18 increase out of scale, increasing the number of release sites and thus transmitter release/quantal content activity even further

and BRP are needed as their loss occludes an increase in Syx-1Aand Unc13A levels (Fig. 4). Somewhat unexpectedly, although AZ-remodeling occurs on the same minutes' time-scale, it is dispensable for the rapid potentiation of NT-release during PhTxinduced PHP (e.g., brp ${ }^{\text {Null, }}$ aplip- $e^{e k 4}$ or $s r p k 79 D^{A T C}$ ) (Fig. 6). However, the remodeling is essential for the long-term consolidation of the potentiation (the capacity to restore the APevoked response in gluRIIA Null mutants was severely impaired when combined with $b r p^{\text {Null }}$ or $s r p k 79 D^{A T C}$ ) (Fig. 6).

Thus, the rapid release enhancement appears to capitalize on AZ-material that is already present, for example by increasing $\mathrm{Ca}^{2+}$ influx ${ }^{44}$ and by the activation of already present but dormant release sites consistent with PHP depending on RIM, RBP, and Unc13A (refs. ${ }^{16,17}$ and this study). Interestingly, the rapid potentiation coincides with the accumulation of BRP, Cac, RBP, Unc13A, Syx-1A, and (later) Unc18 in the AZ, which is required to consolidate and possibly extend the release enhancement. Therefore, the synapse utilizes two coincident programs which together ensure immediate rescue and also supply the synapse with backup-material in the form of $\mathrm{BRP} / \mathrm{RBP} / \mathrm{Unc13A}$ nanomodules in case the disturbance should persist.

Notably, recent work using STED microscopy to characterize hippocampal synapses also identified AZ nano-modules by clusters of Bassoon, vGlut, and Synaptophysin, and observed a scaling of vGlut and Synaptophysin upon chemically induced LTP $^{62}$. This nicely aligns with the structural AZ-remodeling described here, suggesting an evolutionarily conserved process that tunes synaptic transmission by adding nano-modular structures to both sides of the synapse. In addition, activity dependent alterations in Syx-1A nano-clusters were also recently described $^{63}$, further pointing to the AZ being a highly dynamic structure which adapts to different environmental demands.

How does the AZ scaffold remodel within minutes? While local protein translation is required for some forms of plasticity ${ }^{64}$, acute translation block did not interfere with PhTx induced AZ- remodeling (Supplementary Fig. 5a). However, we found evidence that effective $\mathrm{AZ}$ protein transport and a functional cytoskeleton is a precondition here: The $\mathrm{BRP} / \mathrm{RBP}$ transport adaptor/regulator proteins Aplip-1 and Srpk79D were required for rapid enhancement of BRP/RBP AZ levels (and loss of Aplip-1-mediated BRP/ RBP transport impaired short-term memory (Supplementary Fig. 9d, e)). Moreover, acute actin-depolymerization prevented the PhTx-induced BRP/Unc13A addition into AZs, further supporting a crucial role for their transport and in line with a recent study where Drosophila Mical, a highly conserved, multi-domain cytoplasmic protein that mediates actin depolymerization, was shown to be necessary for $\mathrm{PHP}^{59}$.

Considering the short timeframe of this adaptation, long-range transport appears unlikely. Instead, we favor the idea that Aplip-1 and Srpk79D function to engage an AZ-proximal reserve pool of components for rapid integration. This pool could originate from a local reservoir in the distal axon or terminal, or even between AZs, from which plasticity may trigger integration into established $\mathrm{AZs}^{18}$. Transport processes may fill or empty the reservoir. Between AZs, the reservoir could be composed of diffusely distributed proteins falling below the detection limit ${ }^{65}$, or could reflect a local rearrangement of material (note the reduction of AZs containing few AZ-protein modules after PhTx treatment (Fig. 2c-e) or upon gluRIIA ablation (Supplementary Fig. 2a)). Reducing the amount of BRP (by removing one gene copy) blocked the rapid structural adaptation (Fig. 4a, b), possibly because all available material was required to build AZs of proper functionality leaving no material for the reservoir. Regardless of the specific molecular mechanism, guided active transport along the cellular cytoskeleton or rearrangements of the cytoskeleton itself appear to serve a general function in synaptic plasticity in multiple species ${ }^{59,66-68}$.

RIM and RBP are established targets for multiple forms of presynaptic plasticity in several synapse types and species $^{10,16,17,57}$. Here, we additionally identified a critical role for 
Unc13A (Fig. 6). In fact, with our data we can infer the interrelation between these factors in presynaptic plasticity. RIM proteins are known to activate (M)Unc13s in several species $^{46-48,69,70}$. While C. elegans and mouse (M)Unc13 proteins interact via an N-terminal C2A domain with RIM, no such domain is known for Drosophila, but the principal functional interaction may well be conserved (via another region of the $\mathrm{N}$-term). This was directly tested by an Unc13A mutant, whose $\mathrm{N}$-term was deleted ${ }^{7}$. This mutant largely rescued the severe loss of synaptic transmission in the unc $13^{\text {Null }}$ condition with APevoked transmission comparable to the full-length Unc13A rescue (compare black with grey traces in Fig. 6h). However, this mutant completely lacked the capacity to undergo PHP, consistent with a required RIM/RBP/Unc13A interplay for plasticity. Furthermore, expressing the same mutant in the Drosophila memory center, the mushroom body, severely impaired shortterm memory formation (Fig. 7), pointing to a relevance of the RIM/RBP/Unc13 plasticity module in the Drosophila central nervous system.

Thus, the morphological and molecular similarities between long-term sensitization in the famous sea slug Aplysia, presynaptic LTP in the mammalian brain, and homeostatic plasticity or learning in Drosophila indicate that the sequence of molecular events we describe here might be highly conserved.

\section{Methods}

Fly husbandry, stocks, and handling. Fly strains were reared under standard laboratory conditions ${ }^{71}$ and raised at $25^{\circ} \mathrm{C}$ on semi-defined medium (Bloomington recipe). For RNAi experiments flies and larvae were kept at $29^{\circ} \mathrm{C}$. For experiments both male and female third instar larvae or flies were used. The following genotypes were used: Wild-type: $+/+\left(w^{1118}\right)$. gluRIIA Null: $d f(2 L) l^{h 4} / d f(2 L) g l u R I I A \& I I B^{S P 22}$ (A22);GluRIIB-GFP/+ or AD9/df(2 L) cl $l^{h 4}$ or gluRIIA ${ }^{\text {SP16 } / g l u R I I A}{ }^{\text {SP16. Supple- }}$ mentary Fig. 1a: Ok6::Syd-1-GFP: Ok6-Gal4/+;UAS-Syd-1-GFP/+. Transport mutants: Figs. 3, 5; Supplementary Fig. 7: aplip-1 ${ }^{\text {Null: }}$ aplip-1 ${ }^{\text {ex213/Df(3L)BSC799; }}$

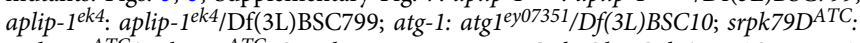
srpk79D $D^{A T C} /$ srpk79D $^{A T C}$. Supplementary Fig. 5c: Ctrl: Ok6-Gal4/+;UAS-Unc13AGFP/+; aplip-1-RNAi: Ok6-Gal4/+; UAS-Unc13A-GFP/UAS-Aplip-1-RNAi; Supplementary Fig. 5d; Ctrl: Ok6-Gal4/+; UAS-Unc13A-GFP/+; srpk79D-RNAi: Ok6Gal4/+; UAS-Unc13A-GFP/UAS-srpk79D-RNAi. Intravital imaging: Movie 1 and Supplementary Fig. 5e-g: Ok6/+; UAS-Unc13A-GFP/UAS-BRP-D3-Straw. Figures 4, 5: $r b p^{\mathrm{Null}}$ : $r b p^{S t o p 1} / r b p^{S 2.01} ; b r p^{\mathrm{Null}}: b r p^{\Delta 6.1} / b r p^{69} ; b r p^{\mathrm{Null} /} / \mathrm{t} b r p^{69} /+$;

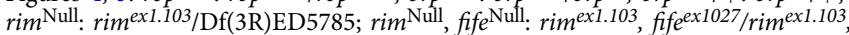
fife $e^{\text {ex1027}}$; liprin- $\alpha^{\text {Null: }}$ liprin- $\alpha^{\text {F3ex15} / l i p r i n-} \alpha^{R 60}$; syd-1 $1^{\text {Null: }}$ syd-1 $1^{1.2} /$ syd-1 $1^{3.4}$. Figure 5 :

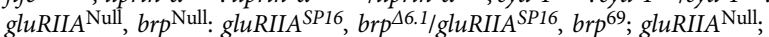
srpk79D ${ }^{A T C}:$ AD9/df(2L)cl $h^{4}$; srpk79D ${ }^{A T C} / \operatorname{srpk} 79 D^{A T C}$. Supplementary Fig. 7 gluRIIA $^{\text {Null }}$;aplip- $1^{e k 4}$ : AD9/df(2L)cl ${ }^{h 4}$;aplip-1 $1^{\text {ek4 }} / D f(3 L) B S C 799$. Figure 6; Supplementary Fig. 8: unc13A $A^{\text {Null: }}$ EMS7.5/P84200; For UAS-Cac-GFP in unc13A Null: Ctrl: Ok6-Gal4, UAS-Cac-GFP/+; unc13A Null: Ok6-Gal4, UAS-Cac-GFP/+;;EMS7.5/ P84200; Unc13A-GFP: elav-GAL4/+;UAS-Unc13A-GFP/+;P84200/P84200; C-term-GFP: elav-GAL4/+;UAS-C-term-GFP/+; P84200/P84200. Learning and memory: Fig. 7; Supplementary fig. 9: Ok107::+: Ok107-Gal4/+; Ok107::Aplip-

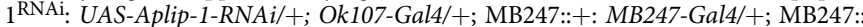
Aplip-1-RNAi: MB247-Gal4/UAS-Aplip-1-RNAi; UAS-Unc13A ${ }^{\mathrm{RNAi}}::+:$ UASUnc13A-RNAi/+; Ok107::UAS-C-term-GFP: UAS-C-term-GFP/+; Ok107-Gal4/+: Ok107::UAS-C-term-GFP::Unc13A RNAi: UAS-C-term-GFP/UAS-Unc13A-RNAi Ok107-Gal4/+; Ok107::UAS-Unc13A RNAi: UAS-Unc13A-RNAi/+; Ok107-Gal4/+. Figure 7b: Ok107::+: VT1211-LexA::LexAop-GCaMP6f/+;;Ok107-Gal4/+; Ok107:: UAS-C-term-GFP: VT1211-LexA::LexAop-GCaMP6f/+;UAS-C-term-GFP/+; Ok107-Gal4/+; Ok107::UAS-C-term-GFP::Unc13A RNAi: VT1211-LexA::LexAopGCaMP6f/+; UAS-C-term-GFP/UAS-Unc13A-RNAi; Ok107-Gal4/+. Western blot, Supplementary Fig. 9a: elav::+: elav-Gal4/+; UAS-Unc13A-RNAi::+: UASUnc13A-RNAi/+; elav::UAS-Unc13A RNAi. elav-Gal4/+;;UAS-Unc13A-RNAi/+. Stocks were obtained from: $A 22^{22}$; $A D 9, d f(2 L) l^{h 4}, g l u R I I A^{S P 16} 23$; GluRIIB-

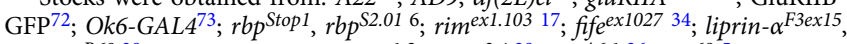
liprin- $\alpha^{R 6038}$; UAS-Syd-1-GFP; syd-1 ${ }^{1.2}$, syd-1 ${ }^{3.439}$; brp ${ }^{\Delta 6.136}$; brp ${ }^{69}$ 5; EMS7.5, UAS-Unc13A-GFP ${ }^{4}$; UAS-BRP-D3-Straw ${ }^{72}$; UAS-Cac-GFP ${ }^{74}$; elav-Gal475; UAS-

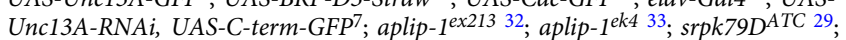
Ok107-Gal4 ${ }^{76}$; MB247-Gal4 ${ }^{77}$. P84200 was provided by the Drosophila Genetic Resource Center (DGRC). The aplip-1 ${ }^{e k 4} ; \mathrm{Df}(3 \mathrm{~L}) \mathrm{BSC799}$; $\operatorname{atg}^{\text {ey07351; }}$ Df(3L) BSC10; Df(3R)ED5785 lines were provided by the Bloomington Drosophila Stock Center. UAS-Aplip-1-RNAi and UAS-srpk79D-RNAi from VDRC.

Immunostaining. Larvae were dissected and stained as described previously ${ }^{39}$. The following primary antibodies were used: guinea-pig Unc13A $\left(1: 500^{4}\right)$; mouse Syx1A
8C3 (1:40; Developmental Studies Hybridoma Bank, University of Iowa, Iowa City, IA, USA; AB Registry ID: AB_528484); mouse Unc18/Rop 4F8 (1:500; Developmental Studies Hybridoma Bank, University of Iowa, Iowa City, IA, USA; AB Registry ID: AB_1157869; mouse GFP 3E6 (1:500, Thermo Fisher Scientific Inc., MA, USA, A-11120; AB Registry ID: AB 221568), mouse Nc82=anti-BRPC-term (1:100, Developmental Studies Hybridoma Bank, University of Iowa, Iowa City, IA, USA; AB Registry ID: AB_2314865); rabbit BRPLast200 (1:1000 $\left.{ }^{78}\right)$; rabbit RBPC-term $\left(1: 500^{6}\right)$; guinea-pig vGlut; $\left(1: 2000^{79}\right)$. Except for staining against RBP, Syx1A and Unc18, where larvae were fixed for $10 \mathrm{~min}$ with $4 \%$ paraformaldehyde (PFA) in 0.1 $\mathrm{mM}$ phosphate buffered saline (PBS), all fixations were performed for $5 \mathrm{~min}$ with ice-cold methanol. The glutamate receptor blocker PhTx-433 (Sigmal-Aldrich, MO USA) was prepared as a $4 \mathrm{mM}$ stock solution either in DMSO (final DMSO concentration, $0.5 \%$ ) or $\mathrm{dH}_{2} \mathrm{O}$. Rapid pharmacological homeostatic challenge was assessed by incubating semi-intact preparations in $20 \mu \mathrm{M}$ PhTx diluted in HL3 (see below) containing 0 or $1.5 \mathrm{mM} \mathrm{CaCl}_{2}$ for $10 \mathrm{~min}$ at room temperature ${ }^{19}$. Controls were treated in the same way but were incubated either in pure HL3 or HL3 containing 0.5\% DMSO (in dependence of how PhTx stock solution was prepared) for $10 \mathrm{~min}$. After incubation, the dissection was completed and the preparation was rinsed three times with fixative solution. During the dissection, extreme care was taken to avoid excessive stretching of body wall muscles, as this may significantly impair induction of homeostasis ${ }^{19}$.

For translation block experiments semi-intact preparations were pre-incubated in HL3 containing $50 \mu \mathrm{g} / \mathrm{ml}$ Cycloheximide (Chx; Sigmal-Aldrich, MO, USA) dissolved in DMSO for $10 \mathrm{~min}$, a concentration previously shown to block protein synthesis in Drosophila ${ }^{80}$. Rapid pharmacological homeostatic challenge was then assessed by incubating semi-intact preparations in $20 \mu \mathrm{M}$ PhTx (or a similar volume of $\mathrm{H}_{2} \mathrm{O}$ in control experiments) diluted in HL3 (see below) containing $50 \mu \mathrm{g} / \mathrm{ml} \mathrm{Chx}$ for $10 \mathrm{~min}$ at room temperature. For actin-depolymerization experiments semi-intact preparations were pre-incubated in HL3 containing $15 \mu \mathrm{M}$ Latrunculin B (Abcam, UK) in DMSO for $10 \mathrm{~min}$. For control experiments larvae were incubated with a solution containing a similar volume of DMSO. Rapid pharmacological homeostatic challenge was then assessed by incubating semiintact preparations in $20 \mu \mathrm{M}$ PhTx (or a similar volume of $\mathrm{H}_{2} \mathrm{O}$ in control experiments) diluted in HL3 (see below) containing $15 \mu \mathrm{M}$ Latrunculin B in DMSO (or DMSO alone in control experiments) for $10 \mathrm{~min}$ at room temperature. Afterwards, prepping and staining procedures were performed as described above/ below. Control animals were always reared in parallel and treated identically in all experiments.

Secondary antibodies for standard immunostainings were used in the following concentrations: goat anti-HRP-Cy5 (1:250, Jackson ImmunoResearch, PA, USA); goat anti-HRP-647 (1:500, Jackson ImmunoResearch 123-605-021, PA, USA); goat anti-rabbit-Cy3 (1:500, Jackson ImmunoResearch 111-165-144, PA, USA); goat anti-mouse-Cy3 (1:500, Jackson ImmunoResearch 115-165-146); goat antimouse or anti guinea pig Alexa-Fluor-488 (1:500, Life Technologies A11001/ A11073, CA, USA). Larvae were mounted in vectashield (Vector labs, CA, USA). Secondary antibodies for STED were used in the following concentrations: goat anti-mouse or rabbit Alexa594 (1:500, Thermo Fisher Scientific Inc. A11032/ A11037, MA, USA); goat anti-mouse Atto590 (1:100); goat anti-rabbit Atto590 (1:100); goat anti-guinea pig star635 (1:100); goat anti-rabbit star635 (1:100); goat anti-mouse or rabbit Atto647N (1:250; Active Motif; 15038/15048). Atto590 (ATTO-TEC AD 590-31) and star635 (Abberior 1-0101002-1) coupled to respective IgGs (Dianova). For STED imaging larvae were mounted in Mowiol (Max-Planck Institute for Biophysical Chemistry, Group of Stefan Hell) or ProLong Gold (Life-Technologies, CA, USA) on high-precision glass coverslips.

For Western blots ${ }^{81}$, adult flies were dissected in cold Ringer's solution and homogenized in Lysis buffer $(1 \times$ PBS, $0.5 \%$ Triton, $2 \%$ SDS, $1 \times$ Protease inhibitor, $1 \times$ Sample buffer) followed by full-speed centrifugation at $18^{\circ} \mathrm{C}$. One brain's supernatant for each group was subjected to SDS-PAGE and immunoblotted according to standard procedures. The following antibodies were used: guinea-pig Unc13A $\left(1: 2000^{4}\right)$ and mouse Tubulin (1:100,000; Sigmal-Aldrich, MO, USA; Cat\# T9026, AB Registry ID: AB_477593). Antibodies obtained from the Developmental Studies Hybridoma Bank were created by the NICHD of the NIH and maintained at The University of Iowa, Department of Biology, Iowa City, IA 52242.

Image acquisition, processing, and analysis. Confocal microscopy was performed with a Leica SP8 microscope (Leica Microsystems, Germany). Images of fixed and live samples were acquired at room temperature. Confocal imaging of NMJs was done using a $z$-step of $0.25 \mu \mathrm{m}$. The following objective was used: $63 \times 1.4$ NA oil immersion for NMJ confocal imaging. All confocal images were acquired using the LAS X software (Leica Microsystems, Germany). Images from fixed samples were taken from muscle 4 of third instar larval 1b NMJs (segments A2-A5) or nerve bundles (segments A1-A3). Images for figures were processed with ImageJ software to enhance brightness using the brightness/contrast function. If necessary, images were smoothened ( 0.5 pixel Sigma radius) using the Gaussian blur function. Confocal stacks were processed with Image) software (http://rsbweb. nih.gov/ij/). Quantifications of AZs (scored via BRP) were performed following an adjusted manual ${ }^{82}$, briefly as follows. The signal of a HRP-Cy5 antibody was used as template for a mask, restricting the quantified area to the shape of the NMJ. The original confocal stacks were converted to maximal projections, and after background subtraction, a mask of the synaptic area was created by applying a certain 
threshold to remove the irrelevant lower intensity pixels. The segmentation of single spots was done semi-automatically via the command "Find Maxima" embedded in the ImageJ software and by hand with the pencil tool and a line thickness of 1 pixel. To remove high frequency noise a Gaussian blur filter (0.5 pixel Sigma radius) was applied. The processed picture was then transformed into a binary mask using the same lower threshold value as in the first step. This binary mask was then projected onto the original unmodified image using the "min" operation from the ImageJ image calculator. For spots $/ \mu \mathrm{m}^{2}$ the number of spots was divided by the size of the mask of the synaptic area.

For colocalization analysis (Pearson correlation coefficient) the ImageJ plugin "JACOP" (http://rsb.info.nih.gov/ij/plugins/track/jacop2.html) was used. To determine the synaptic protein levels, a custom-written ImageJ script was used that detects the locations with highest local maxima in pixel values to generate regions of interests (ROIs) and sets a point selection at each. The intensities were then measured and all selections were deleted, leaving intensity values and $(x, y)$ locations in the results list. The results list was then used to create a circle of size $=$ 5 pixels (pixel size $100 \mathrm{~nm}$ ) centered around each $(x, y)$ location and the integrated density within these ROIs was measured and taken for further calculations. The same ROIs were then used in the channel containing signals of the co-stained protein. For scatter plots, co-stainings of BRP either with RBP, Unc13A or Syx-1A with or without PhTx-treatment or wild-type and gluRIIANull were used. The AZ numbers were counted (number of BRP spots) and the local synaptic levels of both co-stained proteins were measured. AZs were then sorted into five bins (AZ number divided by five) depending on their synaptic BRP levels and then the respective second channel intensities distributed to the appropriate bin. Binned BRP levels were then plotted against binned levels of the second channel.

STED microscopy. Two-color STED images were recorded on custom-built STEDmicroscopes ${ }^{83,84}$, which either combine two pairs of excitation laser beams of $595 \mathrm{~nm}$ and $635 \mathrm{~nm}$ or $595 \mathrm{~nm}$ and $640 \mathrm{~nm}$ wavelength with one STED fiber laser beam at $775 \mathrm{~nm}$. All STED images were acquired using Imspector Software (Max Planck Innovation GmbH, Germany). STED images were processed using a linear deconvolution function integrated into Imspector Software (Max Planck Innovation $\mathrm{GmbH}$, Germany). Regularization parameters ranged from $1 \mathrm{e}^{-09}$ to $1 \mathrm{e}^{-10}$. The point spread function (PSF) for deconvolution was generated by using a $2 \mathrm{D}$ Lorentz function with its half-width and half-length fitted to the half-width and half-length of each individual image. For Fig. 3c, dual-color STED imaging with time-gated detection was performed using a commercial Leica SP8 TCS STED microscope (Leica Microsystems, Germany) equipped with a $100 \times$ NA 1.4 objective (HC PL Apo CS2; Leica Microsystems, Germany). Briefly, the system includes an inverted DMi8 CS microscope equipped with a 100× pulsed white light laser (WLL; $\sim 80$-ps pulse width, $80-\mathrm{MHz}$ repetition rate; NKT Photonics, Denmark) with a STED lasers for depletion (pulsed) at $775 \mathrm{~nm}$. Detection of Alexa594 after excitation at $594 \mathrm{~nm}$ and emission detection of $604-650 \mathrm{~nm}$ and Atto647 after excitation at $640 \mathrm{~nm}$ at emission of $656-751 \mathrm{~nm}$ was performed in frame sequential mode. Time-gated detection with Hybrid detectors was set from 0.3-6 ns for both dyes. Raw data were deconvolved with Huygens Professional software (Scientific Volume Imaging) using a theoretical PSF automatically computed based on pulsedwave STED optimized function and the specific microscope parameters. Default deconvolution settings were applied. Images for figures and for finding highintensity clusters (see below) were processed with ImageJ software to remove obvious background or neighboring AZs (if required), enhance brightness/contrast and smoothened ( 0.5 pixel Sigma radius) using the Gauss blur function.

Classification and alignment of single AZs. All analysis described below was done using MATLAB R2016b (Mathworks Inc., Natick MA, USA) with optional toolboxes, which are indicated in the respective sections. Classification of individual AZs was achieved by using a custom script to detect the position of cluster centers (local intensity maxima) in the images where speckles of neighboring AZs were removed (only for Unc13A, BRP, RBP), while the averaging procedure described hereafter was performed on the corresponding raw images. This code retains only pixels above a defined grey value threshold. For the analysis of Unc13A stainings shown in Fig. 2f, a threshold value of 25 was used (18 for BRP, RBP, Syx$1 \mathrm{~A}$, and Unc-18). All pixel values in the image below this threshold value were set to zero to remove background noise. We then identified the positions of highintensity pixel clusters as local intensity maxima in the images. This was achieved by finding local maxima in the vertical and horizontal pixel lines. First, the function searched the first derivative (using the function diff) of all pixel columns for zero values and the changes in the surrounding slopes were considered to identify local maxima. The same procedure was then applied in pixel rows, but only for those pixel column values associated with a local maximum in the previous step. All single pixels that were associated with a maximum in both a row and a column were detected using the function intersect. To prevent detection of the same cluster more than once, a defined minimum distance of clusters was used ( $50 \mathrm{~nm}$ for BRP, Unc13A, Syx-1A and $20 \mathrm{~nm}$ for RBP) and only the local maximum with the highest intensity value was considered. All subsequent translation and averaging procedures were performed on corresponding raw images of the same AZs using the determined classification and positions of clusters. The non-cleaned AZs were sorted by the number of protein clusters detected this way. To calculate the center of mass of all coordinates, the means of all $x$ - and $y$-coordinates were taken according to equations (1) and (2)

$$
\begin{aligned}
& \mathrm{Sx}=n^{-1} \cdot \sum_{1}^{n} x_{\mathrm{obs}}(n) \\
& \mathrm{Sy}=n^{-1} \cdot \sum_{1}^{n} y_{\mathrm{obs}}(n)
\end{aligned}
$$

where $(\mathrm{Sx}, \mathrm{Sy})$ is the $(x, y)$-coordinate center of mass in the initial image, $n$ is the number of identified clusters, and $x_{\mathrm{obs}}(n)$ and $y_{\mathrm{obs}}(n)$ are the positions of the $n$-th cluster in the present image. To align the center of mass to the center of the image, the necessary shift ( $\Delta x$ and $\Delta y$ ) of the original coordinates was calculated according to Eqs. (3) and (4) and used subsequently in Eqs. (5) and (6).

$$
\begin{aligned}
& \Delta x=0.5 \cdot \operatorname{imsize}(x)-\mathrm{Sx} \\
& \Delta y=0.5 \cdot \operatorname{imsize}(y)-\mathrm{Sy} \\
& x_{\text {centered }}(n)=x_{\mathrm{obs}}(n)+\Delta x \\
& y_{\text {centered }}(n)=y_{\text {obs }}(n)+\Delta y
\end{aligned}
$$

In Eqs. (3) and (4), "imsize" refers to the size of the image in $x$ or $y$ dimension. The resulting coordinates $x_{\text {centered }}$ and $y_{\text {centered }}$ represent cluster coordinates after shifting the original coordinates $x_{\mathrm{obs}}$ and $y_{\mathrm{obs}}$. The same translation was applied to the corresponding AZ image (using the function imtranslate, part of the 'Image Processing' toolbox). Clusters were ranked in a counter-clockwise sequence in all images (see illustration in Fig. 2b; Supplementary Fig. 3c) by sorting them for increasing angle between the image center and the cluster location in relation to the vertical midline $(x=26)$

To align protein cluster coordinates between all investigated AZs, central rotation (around the image center, $x_{\text {middle }}=y_{\text {middle }}=26$ ), was done using the operation in Eq. (7).

$$
\left(\begin{array}{l}
x_{\text {rotated }}(n) \\
y_{\text {rotated }}(n)
\end{array}\right)=\left[\left(\begin{array}{l}
x_{\text {centered }}(n) \\
y_{\text {centered }}(n)
\end{array}\right)-\left(\begin{array}{l}
x_{\text {middle }} \\
y_{\text {middle }}
\end{array}\right)\right] \cdot\left(\begin{array}{cc}
\cos (\alpha) & -\sin (\alpha) \\
\sin (\alpha) & \cos (\alpha)
\end{array}\right)+\left(\begin{array}{l}
x_{\text {middle }} \\
y_{\text {middle }}
\end{array}\right)
$$

To find the optimal angle to overlay all AZs, a cost reflecting the sum of distances between cluster positions of the same rank in all images was minimized. The cost function was defined as described in Eq. (8).

$$
\begin{array}{r}
\text { cost }=\sum_{n=1}^{\text {totClusters }} \sum_{m=1}^{\text {totImgs }} \sum_{l=1}^{\text {totImgs }}\left(\left(x_{\text {rotated }}(n, m)-x_{\text {rotated }}(n, l)\right)^{2}\right. \\
\left.+\left(y_{\text {rotated }}(n, m)-y_{\text {rotated }}(n, l)\right)^{2}\right)
\end{array}
$$

In Eq. (8), $n$ is the cluster number, totClusters is the total cluster number of the respective category of images, $m$ and $l$ are particular AZ images in the stack of images from one category, and totImgs is the total number of $\mathrm{AZ}$ images in that category. The squared Euclidean distances were calculated using the function pdist, which performs the operation shown in Eq. (8).

The optimal rotation angle was found using a genetic algorithm function (ga, part of the 'Global Optimization' toolbox). The rotation angles evaluated were constrained in a range from -180 to 180 degrees. For faster optimization, parallelization (setting the option 'UseParallel' to 'true'; this requires the 'Parallel Computing' toolbox) was employed to evaluate 500 individual cost functions per generation. Cluster coordinates from all images in one category were rotated simultaneously. A single individual in the genetic algorithm represented a set of rotation angles for each image. The convergence criterion (TolFun) was left at the default value (a relative cost value change of $<10^{6}$ over 50 generations). The output of this optimization was a vector containing all rotation angles that led to the best overlap of cluster coordinates. Finally, these rotations were then applied to the centered original AZ images. All images aligned this way were then combined in a stack and an average image was generated by calculating the mean intensity of all image pixels. For better illustration of the AZ structure, pixel intensities were linearly scaled such that the highest intensity pixel had a value of 255 . The procedure was only performed if more than two images existed in the same cluster number class for at least 5 consecutive cluster number classes. The histograms shown in Fig. 2c-e and Supplementary Fig. 2a were generated by counting the number of images in each cluster number class, and dividing each value by the total amount of images detected in all classes. The mean cluster intensity shown in Supplementary Fig. 2b, c was calculated by taking the average image intensities per category and subtracting the mean intensity of each category from the next higher one. These differences in mean intensity between subsequent categories were then further averaged.

To investigate the AZ structure in an approach independent of the clusterdistance minimization procedure described above, we repeated the averaging in a different way as follows. We developed a MATLAB code for AZ centering and alignment by rotation of the highest intensity pixel to identical angles and therefore 
similar positions, which yielded qualitatively similar results (Supplementary Fig. 3a). Again, high-intensity clusters were detected in AZ images cleaned from clusters belonging to bordering AZs, and all translations and rotations were then performed on unretouched images. The center of mass of the found cluster coordinates was calculated and the image shifted so that the center of mass of the coordinates was in the center of the 51 by 51 pixel space ( $x=y=26$; see Eqs. (1) to (6)). Only the position of the brightest pixel was then considered for rotation. To determine the angle by which to rotate each image to place this highest intensity pixel to the same fixed position (on the vertical midline between the two top image quadrants-the "twelve o'clock" position), the $x$ and $y$ distances to the center of rotation (equal to the center pixel of the image $x=y=26$ ) were calculated to find the length $(l)$ of the hypotenuse and the opposite side of the right triangle using pdist. The angle $\alpha$ in degrees was then calculated by taking the inverse sine in degrees of this value (MATLAB function asind), as shown in Eq. (9).

$$
\alpha=\sin ^{-1}\left(\frac{l_{\text {opposite side }}}{l_{\text {hypotenuse }}}\right)
$$

In cases where the brightest peak was located above the horizontal midline, the adjacent side of the triangle was the vertical midline. In cases where the brightest peak was located below the horizontal midline, the angle was calculated with the horizontal midline being the adjacent side, and $90^{\circ}$ were added to the final angle value. Additionally, in cases where the brightest peak was located to the right of the vertical midline, the angle was multiplied with -1 . To generate the results shown in Supplementary Fig. 3b, which shows the averaging of AZ images within randomly assigned categories, we generated a number of random category values and then proceeded with the averaging procedure described above. For this, we reproduced the distribution of category values from the $\mathrm{AZ}$ dataset according to the histogram values of the category vector as follows. A histogram of the category vector was generated (MATLAB function histogram) with a bin width of 1 , yielding the absolute amount of AZs per category. A cumulative sum vector was calculated from these histogram values (MATLAB function cumsum). For each position in the category vector, we then chose a random number between 0 and 1 and multiplied it by the number of images. We then found the first position in the cumulative sum vector that was larger than this random value. The position found was equal to the assigned category. This resulted in a randomly assigned category vector with a similar distribution of categories as the original vector.

In vivo live imaging and analysis. In vivo imaging of intact Drosophila larvae was performed as previously described ${ }^{85}$. Briefly, third instar larvae were put into a drop of Voltalef H10S oil (Arkema, Inc., France) within an airtight imaging chamber. Before imaging, the larvae were anaesthetized with 20 short pulses of a desflurane (Baxter, IL, USA) air mixture until the heartbeat completely stopped. For assessing axonal transport, axons immediately after exiting the ventral nerve cord were imaged for $5 \mathrm{~min}$ using timelapse confocal microscopy. Kymographs were plotted using a custom-written ImageJ script.

Induction of homeostatic plasticity and electrophysiology. Third-instar larvae were selected and placed individually on a Sylgard block. Using a very sharp pin, the tail of the larva was pinned between the posterior spiracles, in the absence of solution. The head was pinned, making sure not to stretch the larva, so that the animal was relatively loose between the two pins. A small horizontal incision was made in the dorsal cuticle at the tail with a sharp scissors. The larva was cut vertically from the tail incision in an anterior direction (towards the head), continuing beyond the head pin. Great care was taken not to stretch the cuticle or animal during this process. Forty microliters of a $20 \mu \mathrm{M}$ PhTx in modified hemolymph-like solution ( $\mathrm{HL}^{86}$; composition (in $\mathrm{mM}$ ): $\mathrm{NaCl} 70, \mathrm{KCl} 5, \mathrm{MgCl}_{2}$ $10, \mathrm{NaHCO}_{3} 10$, trehalose 5, sucrose 115, HEPES $5, \mathrm{CaCl}_{2} 0, \mathrm{pH}$ adjusted to 7.2 ) was pipetted into the abdominal cavity with minimal force, making sure to fill the abdomen. After $10 \mathrm{~min}$ incubation, the preparation was completed without rinsing. The cuticle was gently pinned twice on each side (without stretching). The connection of the intestines and trachea to the body at the posterior were cut. Holding the now free ends of the intestines and trachea with a fine forceps, remaining connections were cut moving in an anterior direction (towards the head). The intestines and trachea could then be gently removed without stretching the larva. Finally, the brain was held firmly and slightly raised above the body so that the scissors could be placed underneath to cut the segmental nerves. Care was taken not to touch the underlying muscle and to avoid excessive pulling of the nerves before they were cut. The completed preparation was rinsed 3 times with $\mathrm{PhTx}$-free HL3 ( $0 \mathrm{mM} \mathrm{CaCl}, 10 \mathrm{mM} \mathrm{MgCl}$ ). PhTx-free HL3 solution was used in control treatments. Sylgard blocks were kept separate for PhTx and control treatments and all implements were rinsed after each recording.

The Sylgard block and completed larval preparation was placed in the recording chamber which was filled with $2 \mathrm{ml} \mathrm{HL} 3\left(0.4 \mathrm{mM} \mathrm{CaCl}_{2}, 10 \mathrm{mM} \mathrm{MgCl}_{2}\right)$. Recordings were performed at room temperature $\left(\sim 22^{\circ} \mathrm{C}\right)$ in current clamp mode at muscle 6 in segments A2/A3 as previously described ${ }^{86}$ using an Axon Digidata 1550 A digitizer, Axoclamp 900 A amplifier with HS-9A x0.1 headstage (Molecular Devices, CA, USA) and on a BX51WI Olympus microscope with a 40X LUMPlanFL/IR water immersion objective. Sharp intracellular recording electrodes were pulled using a Flaming Brown Model P-97 micropipette puller (Sutter
Instrument, CA, USA) with a resistance of $20-35 \mathrm{M} \Omega$, back-filled with $3 \mathrm{M} \mathrm{KCl}$. Cells were only considered with a membrane potential less than $-60 \mathrm{mV}$ and membrane resistances greater than $4 \mathrm{M} \Omega$. All recordings were acquired using Clampex software (v10.5) and sampled at $10-50 \mathrm{kHz}$, filtering with a $5 \mathrm{kHz}$ lowpass filter. mEPSPs were recorded for $1 \mathrm{~min}$. eEPSPs were recorded by stimulating the appropriate nerve at $0.1 \mathrm{~Hz}$, five times $(8 \mathrm{~V}, 300 \mu$ s pulse) using an ISO-STIM 01D stimulator (NPI Electronic, Germany). Stimulating suction electrodes were pulled on a DMZ-Universal Puller (Zeitz-Instruments GmbH, Germany) and fire polished using a CPM-2 microforge (ALA Scientific, NY, USA). A maximum of two cells were recorded per animal.

Analysis was performed with Clampfit 10.5 and Graphpad Prism 6 software. mEPSPs were further filtered with a $500 \mathrm{~Hz}$ Gaussian low-pass filter. Using a single template for all cells, mEPSPs were identified and analyzed, noting the mean mEPSP amplitude per cell. For Fig. 5 and Supplementary Fig. 7, templates were generated for each cell and the first 30 mEPSPs were identified and taken into account for further analysis. An average trace was generated from the 5 eEPSP traces per cell. The amplitude of the average eEPSP trace was divided by the mean mEPSP amplitude, for each respective cell, to determine the quantal content.

Dissection and current clamp recordings of w1118 vs gluRIIA ${ }^{\text {Null }}$ were performed as above in male third-instar larvae. Cells with an initial membrane potential greater than $-55 \mathrm{mV}$, resistances less than $5 \mathrm{M} \Omega$ or multiple responses to a single stimulus were rejected. eEPSPs were recorded by stimulating the appropriate nerve at $0.2 \mathrm{~Hz}, 10$ times $(6 \mathrm{~V}, 300 \mu$ s pulse). An average eEPSP amplitude was calculated from the 10 traces. mEPSPs were analyzed with a genotype specific template. Quantal contents were calculated by dividing the mean eEPSP by mean mEPSP for each cell.

In vivo two-photon live calcium imaging and analysis. Two-photon imaging of odor-evoked calcium responses was conducted in 3-5-day-old mixed-sex flies expressing LexAop-GCaMP6f in VT1211-LexA. For imaging, flies were briefly anesthetized on ice and mounted in a custom made chamber by immobilizing wings, head and proboscis with wax. The head capsule was opened in sugar-free HL3-like extracellular saline ${ }^{87}$. Odor stimulation consisted of a $1.5 \mathrm{~s}$ OCT pulse followed by a $30 \mathrm{~s}$ break and then a $1.5 \mathrm{~s} \mathrm{MCH}$ pulse followed again by another $30 \mathrm{~s}$ break. This alternating odor pulse protocol was consecutively repeated five times (odor dilution in mineral oil 1/1000). Odors were delivered on a clean air carrier stream and image acquisition and odor stimulation was synchronized temporally using a custom-designed system. Fluorescence was centered on $910 \mathrm{~nm}$ generated by a Ti-Sapphire laser (Chameleon Ultra II, Coherent, CA, USA). Images with a pixel size of $0.3 \times 0.3 \mu \mathrm{m}$ were acquired at $70 \mathrm{~Hz}$ using two-photon microscopy (Femto2D-Resonant by Femtonics Ltd., Hungary) with a $20 \times, 1.0$ NA waterimmersion objective, controlled by MESc v3.5 software (Femtonics Ltd., Hungary). For each animal, a single hemisphere was analyzed. All OCT and MCH responses of a fly were averaged respectively and resulting traces were averaged between flies Mean intensity values of two-photon fluorescence were calculated, while F0 was defined as the mean $\mathrm{F}$ from 0 to $1.5 \mathrm{~s}$ at the beginning of a recording (MESc $\mathrm{v}$ 3.5 software). Image processing for single frames were manually performed using ImageJ (images did not require registration).

Odor avoidance conditioning. All flies were 3 to 5 days old, raised in $12 \mathrm{~h}: 12 \mathrm{~h}$, light:dark cycle and at $65 \%$ relative humidity. One day before the experiment, the flies were transferred to fresh food vials. One hour prior to the experiment, flies were pre-conditioned to experimental conditions (dim red light, $25^{\circ} \mathrm{C}$, humidity of $80 \%$ ). The aversive odors 3-Octanol (OCT) and Methylcyclohexanol (MCH) were diluted 1:100 in paraffin oil and presented in $14 \mathrm{~mm}$ cups. A current of $120 \mathrm{~V}$ AC was used as a behavioral reinforcer. The associative training was performed as previously described ${ }^{88}$. In a single-cycle training, nearly 100 flies were presented with one odor $\left(\mathrm{CS}^{+}\right)$paired with electrical shock (US; 12 times for $1 \mathrm{~min}$ ). After one minute of pure air-flow, the second odor was presented without the shock $\left(\mathrm{CS}^{-}\right)$for another minute. The flies were then immediately tested for short-term memory performance by presenting them the two odors together. A performance index (PI) was calculated as the number of flies choosing the odor without shock $\left(\mathrm{CS}^{-}\right)$, minus the number of flies choosing the odor paired with shock $\left(\mathrm{CS}^{+}\right)$, divided by the total number of flies, multiplied by 100 . The values of PI ranges from 0 to 100 where 0 means no learning (50:50 distribution of flies) and a value of 100 means complete learning (all flies avoided the conditioned odor). The final learning index was calculated as the average of both reciprocal indices for the two odors. Odor Avoidance experiments were used to test innate behavior where each odor was presented to the flies without conditioning. The PIs were calculated as stated above.

Quantification and statistical analysis. Data were analyzed using Prism (GraphPad Software, CA, USA). Per default Student's $T$ test was performed to compare the means of two groups unless the data were either non-normally distributed (as assessed by D'Agostino-Pearson omnibus normality test) or if variances were unequal (assessed by $F$ test) in which case they were compared by a Mann-Whitney $U$ Test. However, in the Source Data file both tests are provided for all relevant cases. For comparison of more than two groups, one-way analysis of variance (ANOVA) tests were used, followed by a Tukey's multiple comparison test. $P$ values and $\mathrm{N}$ values are 
given in the Source Data file. Means are annotated \pm s.e.m. Asterisks are used to denote significance: ${ }^{\star} P<0.05 ;{ }^{* *} P<0.01 ;{ }^{* *} P<0.001$; n.s. (not significant), $P>0.05$.

Reporting summary. Further information on experimental design is available in the Nature Research Reporting Summary linked to this article.

\section{Data availability}

The data that support the findings of this study, additional information and requests for resources and reagents as well as MATLAB and ImageJ codes used in this study are available from Alexander M. Walter (awalter@fmp-berlin.de) upon request.

Received: 10 August 2018 Accepted: 7 February 2019

Published online: 06 March 2019

\section{References}

1. Citri, A. \& Malenka, R. C. Synaptic plasticity: multiple forms, functions, and mechanisms. Neuropsychopharmacology. 33, 18-41 (2008).

2. Nicoll, R. A. \& Roche, K. W. Long-term potentiation: peeling the onion. Neuropharmacology 74, 18-22 (2013)

3. Acuna, C., Liu, X. \& Sudhof, T. C. How to make an active zone: unexpected universal functional redundancy between RIMs and RIM-BPs. Neuron 91, 792-807 (2016)

4. Bohme, M. A. et al. Active zone scaffolds differentially accumulate Unc13 isoforms to tune $\mathrm{Ca}(2+)$ channel-vesicle coupling. Nat. Neurosci. 19, 1311-1320 (2016).

5. Kittel, R. J. et al. Bruchpilot promotes active zone assembly, Ca2+ channel clustering, and vesicle release. Science 312, 1051-1054 (2006)

6. Liu, K. S. et al. RIM-binding protein, a central part of the active zone, is essential for neurotransmitter release. Science 334, 1565-1569 (2011).

7. Reddy-Alla, S. et al. Stable positioning of Unc13 restricts synaptic vesicle fusion to defined release sites to promote synchronous neurotransmission. Neuron 95, 1350-1364 e1312. (2017)

8. Sakamoto, H. et al. Synaptic weight set by Munc13-1 supramolecular assemblies. Nat. Neurosci. 21, 41-49 (2018).

9. Sudhof, T. C. The presynaptic active zone. Neuron 75, 11-25 (2012).

10. Tang, A. H. et al. A trans-synaptic nanocolumn aligns neurotransmitte release to receptors. Nature 536, 210-214 (2016).

11. Walter, A. M., Bohme, M. A. \& Sigrist, S. J. Vesicle release site organization at synaptic active zones. Neurosci. Res. 127, 3-13 (2018).

12. Wang, S. S. et al. Fusion competent synaptic vesicles persist upon active zone disruption and loss of vesicle docking. Neuron 91, 777-791 (2016).

13. Davis, G. W. \& Muller, M. Homeostatic control of presynaptic neurotransmitter release. Annu. Rev. Physiol. 77, 251-270 (2015).

14. Frank, C. A. Homeostatic plasticity at the Drosophila neuromuscular junction. Neuropharmacology 78, 63-74 (2014)

15. Bruckner, J. J. et al. Fife organizes synaptic vesicles and calcium channels for high-probability neurotransmitter release. J. Cell. Biol. 216, 231-246 (2017).

16. Muller, M., Genc, O. \& Davis, G. W. RIM-binding protein links synaptic homeostasis to the stabilization and replenishment of high release probability vesicles. Neuron 85, 1056-1069 (2015)

17. Muller, M., Liu, K. S., Sigrist, S. J. \& Davis, G. W. RIM controls homeostatic plasticity through modulation of the readily-releasable vesicle pool. $J$ Neurosci. 32, 16574-16585 (2012).

18. Weyhersmuller, A., Hallermann, S., Wagner, N. \& Eilers, J. Rapid active zone remodeling during synaptic plasticity. J. Neurosci. 31, 6041-6052 (2011).

19. Frank, C. A., Kennedy, M. J., Goold, C. P., Marek, K. W. \& Davis, G. W Mechanisms underlying the rapid induction and sustained expression of synaptic homeostasis. Neuron 52, 663-677 (2006).

20. Goel, P., Li, X. \& Dickman, D. Disparate postsynaptic induction mechanisms ultimately converge to drive the retrograde enhancement of presynaptic efficacy. Cell Rep. 21, 2339-2347 (2017).

21. Ortega, J. M., Genc, O. \& Davis, G. W. Molecular mechanisms that stabilize short term synaptic plasticity during presynaptic homeostatic plasticity. Elife 7, e40385 (2018)

22. DiAntonio, A., Petersen, S. A., Heckmann, M. \& Goodman, C. S. Glutamate receptor expression regulates quantal size and quantal content at the Drosophila neuromuscular junction. J. Neurosci. 19, 3023-3032 (1999).

23. Petersen, S. A., Fetter, R. D., Noordermeer, J. N., Goodman, C. S. \& DiAntonio, A. Genetic analysis of glutamate receptors in Drosophila reveals a retrograde signal regulating presynaptic transmitter release. Neuron $\mathbf{1 9}$, 1237-1248 (1997).

24. Ehmann, N. et al. Quantitative super-resolution imaging of Bruchpilot distinguishes active zone states. Nat. Commun. 5, 4650 (2014).
25. Wilhelm, B. G. et al. Composition of isolated synaptic boutons reveals the amounts of vesicle trafficking proteins. Science 344, 1023-1028 (2014).

26. Akins, M. R., Berk-Rauch, H. E. \& Fallon, J. R. Presynaptic translation: stepping out of the postsynaptic shadow. Front. Neural Circuits 3, 17 (2009).

27. Tsurudome, K. et al. The Drosophila miR-310 cluster negatively regulates synaptic strength at the neuromuscular junction. Neuron 68, 879-893 (2010).

28. Wairkar, Y. P. et al. Unc-51 controls active zone density and protein composition by downregulating ERK signaling. J. Neurosci. 29, 517-528 (2009).

29. Johnson, E. L. 3rd, Fetter, R. D. \& Davis, G. W. Negative regulation of active zone assembly by a newly identified SR protein kinase. PLoS Biol. 7, e1000193 (2009).

30. Nieratschker, V. et al. Bruchpilot in ribbon-like axonal agglomerates, behavioral defects, and early death in SRPK79D kinase mutants of Drosophila. PLoS Genet. 5, e1000700 (2009).

31. Siebert, M. et al. A high affinity RIM-binding protein/Aplip1 interaction prevents the formation of ectopic axonal active zones. Elife 4, e06935 (2015).

32. Klinedinst, S., Wang, X., Xiong, X., Haenfler, J. M. \& Collins, C. A. Independent pathways downstream of the Wnd/DLK MAPKKK regulate synaptic structure, axonal transport, and injury signaling. J. Neurosci. 33, 12764-12778 (2013)

33. Horiuchi, D., Barkus, R. V., Pilling, A. D., Gassman, A. \& Saxton, W. M. APLIP1, a kinesin binding JIP-1/JNK scaffold protein, influences the axonal transport of both vesicles and mitochondria in Drosophila. Curr. Biol. 15, 2137-2141 (2005)

34. Bruckner, J. J. et al. Fife, a Drosophila Piccolo-RIM homolog, promotes active zone organization and neurotransmitter release. J. Neurosci. 32, 17048-17058 (2012).

35. Dai, Y. et al. SYD-2 Liprin-alpha organizes presynaptic active zone formation through ELKS. Nat. Neurosci. 9, 1479-1487 (2006).

36. Fouquet, W. et al. Maturation of active zone assembly by Drosophila Bruchpilot. J. Cell. Biol. 186, 129-145 (2009).

37. Hallam, S. J., Goncharov, A., McEwen, J., Baran, R. \& Jin, Y. SYD-1, a presynaptic protein with PDZ, C2 and rhoGAP-like domains, specifies axon identity in C. elegans. Nat. Neurosci. 5, 1137-1146 (2002).

38. Kaufmann, N., DeProto, J., Ranjan, R., Wan, H. \& Van Vactor, D. Drosophila liprin-alpha and the receptor phosphatase Dlar control synapse morphogenesis. Neuron 34, 27-38 (2002)

39. Owald, D. et al. A Syd-1 homologue regulates pre- and postsynaptic maturation in Drosophila. J. Cell. Biol. 188, 565-579 (2010).

40. Akbergenova, Y., Cunningham, K. L., Zhang, Y. V., Weiss, S. \& Littleton, J. T. Characterization of developmental and molecular factors underlying release heterogeneity at Drosophila synapses. Elife 7, e38268 (2018).

41. Holderith, N. et al. Release probability of hippocampal glutamatergic terminals scales with the size of the active zone. Nat. Neurosci. 15, 988-997 (2012).

42. Muhammad, K. et al. Presynaptic spinophilin tunes neurexin signalling to control active zone architecture and function. Nat. Commun. 6, 8362 (2015).

43. Peled, E. S. \& Isacoff, E. Y. Optical quantal analysis of synaptic transmission in wild-type and rab3-mutant Drosophila motor axons. Nat. Neurosci. 14, 519-526 (2011)

44. Muller, M. \& Davis, G. W. Transsynaptic control of presynaptic $\mathrm{Ca}(2)(+)$ influx achieves homeostatic potentiation of neurotransmitter release. Curr. Biol. 22, 1102-1108 (2012).

45. Gratz, S. J. et al. Endogenous tagging reveals differential regulation of $\mathrm{Ca} 2+$ channels at single AZs during presynaptic homeostatic potentiation and depression. J. Neurosci. Preprint at: https://doi.org/10.1523/JNEUROSCI.306818.2019 (2019).

46. Camacho, M. et al. Heterodimerization of Munc13 C2A domain with RIM regulates synaptic vesicle docking and priming. Nat. Commun. 8, 15293 (2017)

47. Deng, L., Kaeser, P. S., Xu, W. \& Sudhof, T. C. RIM proteins activate vesicle priming by reversing autoinhibitory homodimerization of Munc13. Neuron 69, 317-331 (2011)

48. Dulubova, I. et al. A Munc13/RIM/Rab3 tripartite complex: from priming to plasticity? EMBO J. 24, 2839-2850 (2005).

49. Hu, Z., Tong, X. J. \& Kaplan, J. M. UNC-13L, UNC-13S, and Tomosyn form a protein code for fast and slow neurotransmitter release in Caenorhabditis elegans. eLife 2, e00967 (2013).

50. Zhou, K., Stawicki, T. M., Goncharov, A. \& Jin, Y. Position of UNC-13 in the active zone regulates synaptic vesicle release probability and release kinetics. eLife 2, e01180 (2013)

51. Kandel, E. R., Dudai, Y. \& Mayford, M. R. The molecular and systems biology of memory. Cell 157, 163-186 (2014).

52. Krashes, M. J., Keene, A. C., Leung, B., Armstrong, J. D. \& Waddell, S. Sequential use of mushroom body neuron subsets during drosophila odor memory processing. Neuron 53, 103-115 (2007).

53. Aso, Y. et al. The neuronal architecture of the mushroom body provides a logic for associative learning. eLife 3, e04577 (2014). 
54. Owald, D. et al. Activity of defined mushroom body output neurons underlies learned olfactory behavior in Drosophila. Neuron 86, 417-427 (2015).

55. Quinn, W. G. \& Dudai, Y. Memory phases in Drosophila. Nature 262, 576-577 (1976).

56. Takeuchi, T., Duszkiewicz, A. J. \& Morris, R. G. The synaptic plasticity and memory hypothesis: encoding, storage and persistence. Philos. Trans. R. Soc. Lond. B. Biol. Sci. 369, 20130288 (2014).

57. Castillo, P. E. Presynaptic LTP and LTD of excitatory and inhibitory synapses. Cold Spring Harb. Perspect. Biol. 4, a005728 (2012).

58. Newman, Z. L. et al. Input-Specific Plasticity and Homeostasis at the Drosophila Larval Neuromuscular Junction. Neuron 93, 1388-1404 e1310 (2017).

59. Orr, B. O., Fetter, R. D. \& Davis, G. W. Retrograde semaphorin-plexin signalling drives homeostatic synaptic plasticity. Nature 550, 109-113 (2017).

60. Wentzel, C., Delvendahl, I., Sydlik, S., Georgiev, O. \& Muller, M. Dysbindin links presynaptic proteasome function to homeostatic recruitment of low release probability vesicles. Nat. Commun. 9, 267 (2018).

61. Li, X. et al. Synapse-specific and compartmentalized expression of presynaptic homeostatic potentiation. Elife 7, e34338 (2018).

62. Hruska, M., Henderson, N., Le Marchand, S. J., Jafri, H. \& Dalva, M. B. Synaptic nanomodules underlie the organization and plasticity of spine synapses. Nat. Neurosci. 21, 671-682 (2018).

63. Bademosi, A. T. et al. In vivo single-molecule imaging of syntaxin1A reveals polyphosphoinositide- and activity-dependent trapping in presynaptic nanoclusters. Nat. Commun. 8, 13660 (2017).

64. Sutton, M. A. \& Schuman, E. M. Dendritic protein synthesis, synaptic plasticity, and memory. Cell 127, 49-58 (2006).

65. Ziv, N. E. Maintaining the active zone: Demand, supply and disposal of core active zone proteins. Neurosci. Res. 127, 70-77 (2018).

66. Puthanveettil, S. V. et al. A new component in synaptic plasticity: upregulation of kinesin in the neurons of the gill-withdrawal reflex. Cell 135, 960-973 (2008).

67. Sugie, A. et al. Molecular Remodeling of the Presynaptic Active Zone of Drosophila Photoreceptors via Activity-Dependent Feedback. Neuron 86, 711-725 (2015).

68. Wang, Z. et al. Myosin $\mathrm{Vb}$ mobilizes recycling endosomes and AMPA receptors for postsynaptic plasticity. Cell 135, 535-548 (2008).

69. Lu, J. et al. Structural basis for a Munc13-1 homodimer to Munc13-1/RIM heterodimer switch. PLoS Biol. 4, e192 (2006).

70. Michelassi, F., Liu, H., Hu, Z. \& Dittman, J. S. A C1-C2 Module in Munc13 Inhibits Calcium-Dependent Neurotransmitter Release. Neuron 95, 577-590 (2017). e575.

71. Sigrist, S. J., Reiff, D. F., Thiel, P. R., Steinert, J. R. \& Schuster, C. M. Experience-dependent strengthening of Drosophila neuromuscular junctions. J. Neurosci. 23, 6546-6556 (2003).

72. Schmid, A. et al. Activity-dependent site-specific changes of glutamate receptor composition in vivo. Nat. Neurosci. 11, 659-666 (2008)

73. Aberle, H. et al. wishful thinking encodes a BMP type II receptor that regulates synaptic growth in Drosophila. Neuron 33, 545-558 (2002).

74. Kawasaki, F., Zou, B., Xu, X. \& Ordway, R. W. Active zone localization of presynaptic calcium channels encoded by the cacophony locus of Drosophila. J. Neurosci. 24, 282-285 (2004).

75. Lin, D. M. \& Goodman, C. S. Ectopic and increased expression of Fasciclin II alters motoneuron growth cone guidance. Neuron 13, 507-523 (1994).

76. Connolly, J. B. et al. Associative learning disrupted by impaired Gs signaling in Drosophila mushroom bodies. Science 274, 2104-2107 (1996).

77. Zars, T., Fischer, M., Schulz, R. \& Heisenberg, M. Localization of a short-term memory in Drosophila. Science 288, 672-675 (2000).

78. Ullrich, A. et al. Dynamical Organization of Syntaxin-1A at the Presynaptic Active Zone. PLoS Comput. Biol. 11, e1004407 (2015).

79. Chen, X. et al. The BLOC-1 subunit pallidin facilitates activity-dependent synaptic vesicle recycling. eNeuro 4, ENEURO.0335-16.2017 (2017).

80. Tully, T., Preat, T., Boynton, S. C. \& Del Vecchio, M. Genetic dissection of consolidated memory in Drosophila. Cell 79, 35-47 (1994).

81. Huang, Y. et al. The glycosphingolipid MacCer promotes synaptic bouton formation in Drosophila by interacting with Wnt. Elife 7, e38183 (2018).

82. Andlauer, T. F. \& Sigrist, S. J. Quantitative analysis of Drosophila larval neuromuscular junction morphology. Cold Spring Harb. Protoc. 2012, 490-493 (2012).

83. Gottfert, F. et al. Strong signal increase in STED fluorescence microscopy by imaging regions of subdiffraction extent. Proc. Natl. Acad. Sci. USA 114, 2125-2130 (2017).

84. Gottfert, F. et al. Coaligned dual-channel STED nanoscopy and molecular diffusion analysis at $20 \mathrm{~nm}$ resolution. Biophys. J. 105, L01-L03 (2013).
85. Fuger, P., Behrends, L. B., Mertel, S., Sigrist, S. J. \& Rasse, T. M. Live imaging of synapse development and measuring protein dynamics using two-color fluorescence recovery after photo-bleaching at Drosophila synapses. Nat. Protoc. 2, 3285-3298 (2007).

86. Zhang, B. \& Stewart, B. Electrophysiological recording from Drosophila larval body-wall muscles. Cold Spring Harb. Protoc. 2010, pdb prot5487 (2010).

87. Yoshihara, M. Simultaneous recording of calcium signals from identified neurons and feeding behavior of Drosophila melanogaster. J. Vis. Exp. 3625 (2012).

88. Tully, T. \& Quinn, W. G. Classical conditioning and retention in normal and mutant Drosophila melanogaster. J. Comp. Physiol. A 157, 263-277 (1985).

89. Barnstedt, O. et al. Memory-relevant mushroom body output synapses are cholinergic. Neuron 89, 1237-1247 (2016).

\section{Acknowledgements}

We thank S. Waddell and Y. Huang for the VT1211-LexA fly line. M.A.B. was supported by the SFB 740. A.T.G. was supported by a NeuroCure Ph.D. fellowship funded by the Deutsche Forschungsgemeinschaft (Exc 257) within the International Graduate Program Medical Neurosciences. M. Jusyte was supported by an Einstein Center for Neurosciences Ph.D. fellowship. U.R. was supported by a fellowship of the International Max Planck Research School by the Max-Planck-Gesellschaft. This work was supported by grants from the Deutsche Forschungsgemeinschaft to S.J.S. (Exc 257, TP A3 and A6 SFB 958, TP B9/SFB665; TP09/SFB740; TRR 186) and A.M.W. (Emmy Noether programme, TRR 186), D.O. (Emmy Noether programme) and from the National Institutes of Health to D. D. (NS091546)

\section{Author contributions}

M.A.B., S.J.S. and A.M.W. conceived the project. M.A.B., A.W.M., C.B.B., P.G. and A.G P. performed fly husbandry and maintenance. M.A.B., M.J., M.L. and P.G. performed confocal and/or STED imaging experiments and M.A.B., P.G. and A.T.G. analyzed the data. A.W.M., M.J. and P.G. performed electrophysiological experiments and analyzed the data. U.R. performed live-imaging experiments and analysis. P.H. provided reagents and suggested additional experiments. A.T.G and A.M.W. developed software codes for image alignment and averaging and A.T.G analyzed the data. P.G. and D.D. conceived and performed translation block experiments. C.B.B. performed adult Drosophila brain antibody staining and imaging. C.B.B. and S.H. performed behavioral experiments and data analysis. S.H. performed Western blots. D.L. and D.O. planned and performed in vivo two-photon live calcium imaging. F.G. and S.W.H. developed and built the STED microscope. M.A.B., S.J.S., and A.M.W. wrote the paper with input from all co-authors.

\section{Additional information}

Supplementary Information accompanies this paper at https://doi.org/10.1038/s41467019-08977-6.

Competing interests: All authors declare no competing intersets.

Reprints and permission information is available online at http://npg.nature.com/ reprintsandpermissions/

Journal Peer Review Information: Nature Communications thanks Frederic Meunier and other anonymous reviewer(s) for their contribution to the peer review of this work. Peer reviewer reports are available.

Publisher's note: Springer Nature remains neutral with regard to jurisdictional claims in published maps and institutional affiliations.

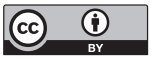

Open Access This article is licensed under a Creative Commons Attribution 4.0 International License, which permits use, sharing, adaptation, distribution and reproduction in any medium or format, as long as you give appropriate credit to the original author(s) and the source, provide a link to the Creative Commons license, and indicate if changes were made. The images or other third party material in this article are included in the article's Creative Commons license, unless indicated otherwise in a credit line to the material. If material is not included in the article's Creative Commons license and your intended use is not permitted by statutory regulation or exceeds the permitted use, you will need to obtain permission directly from the copyright holder. To view a copy of this license, visit http://creativecommons.org/ licenses/by/4.0/

(c) The Author(s) 2019 